\title{
ON THE MEANINGFULNESS OF OPTIMAL SOLUTIONS TO SCHEDULING PROBLEMS: CAN AN OPTIMAL SOLUTION BE NONOPTIMAL?
}

\author{
N. V. R. MAHADEV \\ Northeastern University, Boston, Massachusetts
}

ALEKSANDAR PEKEC̆

University of Aarhus, Aarhus, Denmark

\section{FRED S. ROBERTS}

Rutgers University, New Brunswick, New Jersey

(Received June 1994; revisions received June 1995, August 1996; accepted November 1996)

\begin{abstract}
We consider the problem of finding an optimal schedule for jobs on a single machine when there are penalties for both tardy and early arrivals. We point out that if attention is paid to how these penalties are measured, then a change of scale of measurement might lead to the anomalous situation where a schedule is optimal if these parameters are measured in one way, but not if they are measured in a different way that seems equally acceptable. In particular, we note that if the penalties measure utilities or disutilities, or loss of goodwill or customer satisfaction, then these kinds of anomalies can occur, for instance if we change both unit and zero point in scales measuring these penalties. We investigate situations where problems of these sorts arise for four specific penalty functions under a variety of different assumptions. The results of the paper have implications far beyond the specific scheduling problems we consider, and suggest that considerations of scale of measurement should enter into analysis of conclusions of optimality both in scheduling problems and throughout combinatorial optimization.
\end{abstract}

M any practical problems involve the search for an optimal schedule. We consider the problem of scheduling $n$ jobs on a single machine in which each job has a specified due date or completion time and a penalty is applied for a completion time different from the desired one. In many practical problems, a penalty is applied only for tardy completions; while more generally, a penalty is applied to both early and tardy completions, perhaps in a different way. The interest in scheduling problems where penalties are applied to early arrivals as well as late arrivals is closely tied to the concept of "just-in-time" production, the goal being to have "the right amount of materials of the right quality at the right time in the right place to produce the right quantity of items demanded by the next step of the production" (Cheng 1990). Because penalties can be applied to early arrivals, we allow the machine to lie idle and we schedule without preemption, i.e., we do not allow a job to be interrupted once it is started. The penalties we study involve weighting factors that weight the deviations from desired completion times.

Often these weights are not uniquely determined. For instance, two weight assignments may be equally acceptable if they both give rise to the same ordering of the items or if one is related to the other by a change of scale. Typically weights are measured using some scale of measurement, and we examine the effect on the solution to a scheduling problem if we make admissible changes of scale. We show that in some cases such changes can transform an optimal solution into a nonoptimal one, and we systematically describe those situations when this anomaly occurs. More precisely, we show that the conclusion of optimality can be meaningless in a technical sense that we shall make precise. The main point of this paper is to show that considerations of scale change need to play a role in analysis of scheduling problems. More generally, we wish to make the reader aware of the need to consider sensitivity of conclusions to the change of scales of measurement, not only in the scheduling context, but also in other contexts in operations research. Roberts $(1990,1994)$ makes similar comments about such well-known problems of combinatorial optimization as the shortest path problem and the minimum spanning tree problem, and Cozzens and Roberts (1991) investigate certain graph coloring problems, arising in channel assignments in communications, from a similar point of view.

In the rest of this section, we introduce the basic notation to be used throughout the paper, give some references to the literature, and give a brief overview of the rest of the paper.

We are interested in $n$ jobs in which the $i$ th job has processing time $p_{i}>0$ and desired completion time $d_{i} \geqslant$ 0 . At most one job can be carried out at a time, and there is no preemption. A schedule $S$ (feasible schedule $S$ ) assigns a completion time $C_{i}$ to each job $i$, with time starting 
at 0 and each job $i$ getting processing time $p_{i}$. We let $C(S)$ $=\left(C_{1}, \ldots, C_{n}\right)$ and we also use the vectors $\boldsymbol{d}=\left(d_{1}, \ldots\right.$, $\left.d_{n}\right)$ and $p=\left(p_{1}, \ldots, p_{n}\right)$, where we use the convention that single boldface letters represent vectors. Since no job may start before time 0 , we have $C_{i} \geqslant p_{i}$, for all $i$. Our terminology follows that of Baker and Scudder (1990).

We consider two special cases, those with common due dates, i.e., where $d_{i}=d$ for all $i$, and those with common processing times, i.e., where $p_{i}=p$ for all $i$. The first case is abbreviated cdd, with ncdd standing for the situation where we are not in case cdd. The second case is abbreviated cpt, with ncpt standing for the situation where we are not in case cpt. (It should be noted that in the cdd case, we do not make any assumptions on the common due date $d$. For example, $d$ is not necessarily "large," i.e., we allow $d<$ $\sum_{i=1}^{n} p_{i}$.)

Typically, we have an objective function

$F(S)=F\left(d_{1}, \ldots, d_{n}, C_{1}, \ldots, C_{n}\right)$,

and we seek a (feasible) schedule $S$ that minimizes $F(S)$. Note that the objective function does not explicitly depend upon the processing times. These play a role in determining whether or not a schedule is feasible. We consider only objective functions that depend upon the deviations between desired and actual completion times, and specifically we define the earliness of the $i$ th job

$E_{i}(S)=E_{i}=\max \left\{0, d_{i}-C_{i}\right\}$,

and the tardiness of the $i$ th job

$T_{i}(S)=T_{i}=\max \left\{0, C_{i}-d_{i}\right\}$.

One objective function arises if we simply consider a weighted sum of earliness and tardiness, obtaining

$F(S)=F_{\text {sumE } \mid T}(S)=\sum_{i=1}^{n} \alpha_{i} E_{i}+\sum_{i=1}^{n} \beta_{i} T_{i}$,

where $\alpha_{i}$ and $\beta_{i}$ are positive weights. If only tardiness is penalized, we consider the function

$F(S)=F_{\text {sum } T}(S)=\sum_{i=1}^{n} \beta_{i} T_{i}$

In objective functions $F_{\text {sum } E \mid T}(S)$ and $F_{\text {sumT }}(S)$, we minimize the sum of the deviations. An alternative is to minimize the maximum deviation; we then have the objective functions

$$
\begin{aligned}
& F(S)=F_{\max E \mid T} \\
& \quad=\max \left\{\alpha_{1} E_{1}, \ldots, \alpha_{n} E_{n}, \beta_{1} T_{1}, \ldots, \beta_{n} T_{n}\right\},
\end{aligned}
$$

and

$$
F(S)=F_{\max T}=\max \left\{\beta_{1} T_{1}, \ldots, \beta_{n} T_{n}\right\} .
$$

A variety of other objective functions have been studied in the literature; many are summarized by Baker and Scudder (1990). We limit ourselves to these four. Baker and Scudder (1990) provide motivation for cost functions that are modeled by these objectives.
In the case where $\alpha_{i}=\beta_{i}$ for all $i$, the objective function has symmetric weights and we say that we are in the case of symmetry; we abbreviate this case as s. If we are not in case s, we say we are in case ns. If $\beta_{i}=\beta$ for all $i$ and if $\alpha_{i}$ $=\alpha$ for all $i$ when we are considering earliness penalties, then we say that we have common weights and abbreviate this case as cw. If we do not have common weights, we say we are in case ncw. In Section 2, we systematically study the four penalty functions $F_{\text {sumE|T }}, F_{\text {sum } T}, F_{\max \mid T}$, and $F_{\max T}$ under all combinations of the four assumptions cdd or ncdd, cpt or ncpt, s or ns, and cw or ncw. In other words, we consider sixteen cases for each objective function. Of course, when we do not consider earliness penalties, considerations of symmetry are irrelevant, so there are only eight cases. We make the notational convention that we are in case ns here.

Let us consider the function $F_{\text {sum } E \mid T}(S)$ for example. The case $\mathrm{cw}, \mathrm{cdd}, \mathrm{s}$ (and either $\mathrm{cpt}$ or $\mathrm{ncpt}$ ) gives rise to the objective function

$F(S)=\alpha \sum_{i=1}^{n}\left|C_{i}-d\right|$,

where of course $d_{i}=d$ for all $i$. For all practical purposes, this is the same as the objective function

$F(S)=\sum_{i=1}^{n}\left|C_{i}-d\right|$.

This objective function arises, for example, in the work of Kanet (1981), Sundararaghavan and Admed (1984), Bagchi et al. (1986), Hall (1986), Emmons (1987), Szwarc (1989), and Hall et al. (1991). The case cw, cdd, ns (and cpt or ncpt) gives rise to the objective function

$F(S)=\alpha \sum_{i=1}^{n} E_{i}+\beta \sum_{i=1}^{n} T_{i}$,

and this objective function has been studied by, for example, Panwalkar et al. (1982), Emmons (1987), and Bagchi et al. (1987).

The objective function arising in the case ncw, cdd, ns (and cpt or ncpt) has been studied, for example, by Ahmed and Sundararaghavan (1990), Cheng (1987), Emmons (1987), Quaddus (1987), Bector et al. (1988), Baker and Scudder (1989), Hall and Posner (1991), and Hoogeveen and van de Velde (1991). The objective function arising in the case ncw, ncdd, ns (and cpt or ncpt) has been studied, for example, by Fry et al. (1987a), Fry and Leong (1987), Fry et al. (1987b), Chand and Schneeberger (1988), AbdulRazaq and Potts (1988), and Ow and Morton (1988, 1989). The objective function arising in the case $\mathrm{cw}$, ncdd, $\mathrm{s}$ (and cpt or ncpt) has been studied, for example, by Cheng (1990).

The objective function $F_{\max E \mid T}$ is considered in the work of such authors as Sidney (1977) and, more recently for example, by Ferris and Vlach (1992). The objective functions where only tardiness is considered are studied by a wide variety of authors; two survey papers in this literature 


\section{S122 / Mahadev, Pekec and Roberts}

are that by Koulamas (1994), emphasizing constantweighted tardiness, and that by Abdul-Razaq et al. (1990), concerning weighted tardiness.

In Section 1, we introduce relevant concepts from the theory of measurement and give an example to illustrate how an optimal solution could change to a nonoptimal one under admissible change of scale. Section 2 gives the systematic description of situations when admissible changes of scale preserve optimality. In Section 3, we discuss the results and present issues for further analysis.

\section{MEANINGFULNESS OF THE CONCLUSION OF OPTIMALITY}

In using scales of measurement, we often make somewhat arbitrary choices such as of unit or zero point. One speaks of admissible transformations of scale as transformations of scale values that result from changes in these arbitrary choices. For example, in the case of measurement of mass, we can change from pounds to ounces by changing the unit, and this amounts to multiplying each scale value by $\frac{1}{16}$. Similarly, we can change from kilograms to pounds by multiplying each scale value by approximately 2.2 . When the admissible transformations of scale correspond exactly to multiplication by a positive constant, as here, we say we have a ratio scale. In contrast, if we are measuring temperature, we can change both the unit and the zero point. For example, in going from degrees Centigrade to degrees Fahrenheit, we use the admissible transformation $\frac{9}{5} x+32$, which changes the unit by $\frac{9}{5}$ and the zero point by 32 . When the admissible transformations of scale correspond exactly to multiplication by a positive constant and addition of another constant (not necessarily positive) as here, we say we have an interval scale. When any (strictly) monotone increasing transformation of scale is admissible, we say we have an ordinal scale. An example of an ordinal scale is the Mohs scale of hardness. Every mineral gets a scale value which is an integer between 1 and 10 , and the only significance of the numbers is that a mineral with a higher number scratches a mineral with a lower number. Any (strictly) monotone increasing function of scale values would give a scale with similar properties. Preferences sometimes only give rise to ordinal scales. The theory of scale type was introduced into measurement theory by Stevens $(1946,1951,1959)$. A general introduction to measurement theory can be found in the books by Krantz et al. (1971), Luce et al. (1990), Suppes et al. (1989), and Roberts (1979).

If the truth of a conclusion can depend upon some arbitrary choices involving scales of measurement, as for example about units or zero points, we would probably not want to put much weight behind that conclusion. In measurement theory, we call a statement using scales meaningful if its truth or falsity is unchanged after applying admissible transformations to all of the scales in the statement. We show that in the case of the single machine scheduling problem with earliness and tardiness penalties, the conclu- sion that one schedule is optimal can be meaningless. Whether or not it is meaningful depends upon how we measure penalties.

For further information about meaningful statements, the reader is referred to Roberts $(1985,1994)$ and Luce et al. (1990). For other applications of this concept to problems of combinatorial optimization like the scheduling problems discussed here, see Roberts $(1990,1994)$ and Cozzens and Roberts (1991).

To give a simple example of the application of measurement theoretical ideas to the scheduling problem we have considered, let us suppose that the weights in our objective functions are measured on a ratio scale. This is quite naturally the case, since they are often thought of as dollar penalties, and the dollar defines a ratio scale (we can change the unit by changing to cents or Deutschmarks or Yen). Consider the objective function $F_{\text {sumE|T}}$. Suppose that schedule $S$ is optimal. This means that

$F_{\text {sumE|T }}(S) \leqslant F_{\text {sumE|T }}\left(S^{\prime}\right)$,

for all schedules $S^{\prime}$. For conclusion (1) to be meaningful, we would like it to hold if changes in unit are made in the parameters $\alpha_{i}$ and $\beta_{i}$. Let $F_{\text {sumE } \mid T}^{\prime}\left(S^{\prime}\right)$ denote the value of the objective function for schedule $S^{\prime}$ if $\alpha_{i}$ and $\beta_{i}$ are transformed by a change of scale into $k \alpha_{i}$ and $k \beta_{i}$, respectively, with $k>0$. We want to show that

$F_{\text {sumE } \mid T}^{\prime}(S) \leqslant F_{\text {sumE } \mid T}^{\prime}\left(S^{\prime}\right)$

holds for all schedules $S^{\prime}$. This is true since

$$
\begin{aligned}
F_{\text {sum } E \mid T}^{\prime}\left(S^{\prime}\right) & =\sum_{i=1}^{n} k \alpha_{i} E_{i}\left(S^{\prime}\right)+\sum_{i=1}^{n} k \beta_{i} T_{i}\left(S^{\prime}\right) \\
& =k F_{\text {sum } E \mid T}\left(S^{\prime}\right) .
\end{aligned}
$$

Hence, if weights are measured on a ratio scale, and if objective function $F_{\text {sumE|T }}$ is used, it is meaningful to conclude that a schedule $S$ is optimal. Similar reasoning applies to objective functions $F_{\text {sum } T}(S), F_{\max \mid T}$, and $F_{\max T}$. Hence, we have the following theorem.

Theorem 1.1. Suppose that the objective function is $F_{\text {sumE|T }}, F_{\text {sum } T}(S), F_{\max |T| T}$, or $F_{\max T}$. Further suppose that $\alpha_{i}, \beta_{i}, i=1, \ldots, n$, are measured on a ratio scale. Then the conclusion of optimality is meaningful.

However, if weights are measured on an interval scale, there can be problems, even in the situation where cases cw, cdd, and cpt all apply. To see why, consider the situation where there are three jobs, there are common due dates with $d=3$, and there are common processing times with $p=1$. Suppose that the common weights are given by $\alpha=1$ and $\beta=10$. Note that all three jobs are equivalent since they have equal processing times, desired completion times, and weights. Hence, for any schedule $S^{\prime}$ defined by $C\left(S^{\prime}\right)=\left(C_{1}^{\prime}, C_{2}^{\prime}, C_{3}^{\prime}\right)$, we may assume that $C_{1}^{\prime}<C_{2}^{\prime}<C_{3}^{\prime}$.

Consider the schedule $S$ defined by the vector of completion times $C(S)=(1,2,3)$. For this schedule, 


$$
\begin{aligned}
F_{\text {sumE } \mid T}(S) & =\alpha_{1}(3-1)+\alpha_{2}(3-2)+\beta_{3}(3-3) \\
& =1 \cdot 2+1 \cdot 1+10 \cdot 0=3 .
\end{aligned}
$$

This turns out to be an optimal schedule. In fact $S$ is a unique optimal schedule (up to symmetry). To see why, consider any other schedule $S^{\prime}$ defined by $C\left(S^{\prime}\right)=\left(C_{1}^{\prime}\right.$, $\left.C_{2}^{\prime}, C_{3}^{\prime}\right), C_{1}^{\prime}<C_{2}^{\prime}<C_{3}^{\prime}$. Since processing times are all 1 , we conclude that $C_{1}^{\prime} \geqslant 1, C_{2}^{\prime} \geqslant 2, C_{3}^{\prime} \geqslant 3$. Since $S^{\prime} \neq S$, we must have $C_{3}^{\prime}>3$. If $C_{3}^{\prime} \geqslant 4$, then

$F_{\text {sumE } \mid T}\left(S^{\prime}\right) \geqslant \beta_{3}(4-3)=10 \cdot 1=10>F_{\text {sum } E \mid T}(S)$.

Suppose that $C_{3}^{\prime}<4$. Then because $p_{3}=1, C_{2}^{\prime}<3$. Since $p_{2}=p_{3}=1$, we have $C_{3}^{\prime}-C_{2}^{\prime} \geqslant 1, C_{3}^{\prime}-C_{1}^{\prime} \geqslant 2$. Then, since $C_{3}^{\prime}>3$, we have

$$
\begin{aligned}
F_{\text {sumE|T}}\left(S^{\prime}\right) & =\alpha_{1}\left(3-C_{1}^{\prime}\right)+\alpha_{2}\left(3-C_{2}^{\prime}\right)+\beta_{3}\left(C_{3}^{\prime}-3\right) \\
& =3-C_{1}^{\prime}+3-C_{2}^{\prime}+10\left(C_{3}^{\prime}-3\right) \\
& =8\left(C_{3}^{\prime}-3\right)+\left(C_{3}^{\prime}-C_{2}^{\prime}\right)+\left(C_{3}^{\prime}-C_{1}^{\prime}\right) \\
& \geqslant 8\left(C_{3}^{\prime}-3\right)+1+2>3=F_{\text {sumE } \mid T}(S) .
\end{aligned}
$$

Thus, $S$ is the unique optimal schedule (up to symmetry). Now suppose that we change $\alpha_{i}$ into $k \alpha_{i}+l$ and $\beta_{i}$ into $k \beta_{i}$ $+l$, where $k=1, l=100$ give us a change of unit (no change) and a change of zero point, respectively. We get

$\alpha_{1}^{\prime}=\alpha_{2}^{\prime}=\alpha_{3}^{\prime}=101, \beta_{1}^{\prime}=\beta_{2}^{\prime}=\beta_{3}^{\prime}=110$.

As before, let $F_{\text {sumE|T}}^{\prime}(S)$ denote the value of the objective function for schedule $S$ if $\alpha_{i}$ and $\beta_{i}$ are transformed into $\alpha_{i}^{\prime}$ and $\beta_{i}^{\prime}$, respectively. Then we have

$$
\begin{aligned}
F_{\text {sum } E \mid T}^{\prime}(S) & =\alpha_{1}^{\prime}(3-1)+\alpha_{2}^{\prime}(3-2)+\beta_{3}^{\prime}(3-3) \\
& =101 \cdot 2+101 \cdot 1+110 \cdot 0=303 .
\end{aligned}
$$

Compare $S$ to the schedule $S^{\prime}$ with $C\left(S^{\prime}\right)=(2,3,4)$. We have

$$
\begin{aligned}
F_{\text {sum } E \mid T}^{\prime}\left(S^{\prime}\right) & =\alpha_{1}^{\prime}(3-2)+\beta_{2}^{\prime}(3-3)+\beta_{3}^{\prime}(4-3) \\
& =101 \cdot 1+110 \cdot 0+110 \cdot 1=211 .
\end{aligned}
$$

Thus, schedule $S$ is no longer optimal after a change of unit and zero point in measurement of weights. Hence the conclusion that schedule $S$ is optimal is not meaningful. It does not seem to make sense to assert that a solution to this scheduling problem is optimal if penalties are measured on an interval scale.

Note that it is reasonable in some applications to think of weights as being measured on a scale different from a ratio scale. In particular, if we are not given weights, but only know that some jobs are more important than others, we might only have an ordinal scale. Such a situation often arises in practice. Scheduling problems where problem data are measured on an ordinal scale are discussed by Liu and Sidney (1996a, b) and Liu et al. (1996). Even if weights are thought of as monetary penalties, the scale they define is like an interval scale if we have a fixed fee for currency transactions, e.g., from dollars to Deutschmarks. (Such a transaction is described by the transformation $k x+l$ where $l$ is the fixed fee and where $k$ is the exchange rate, i.e., the amount of Deutschmarks worth one dollar. If we also allow a transformation from dollars to cents, then the fixed fee is 0 .) If the weights measure some value or utility (or disutility) associated with a given early or tardy completion, or loss of goodwill due to completion at a time different than the requested time, then it is possible that we would measure these with a scale where change of zero point as well as change of unit is allowed, as is commonly done in utility theory-see for example Coombs et al. (1970), Krantz et al. (1971), or Roberts (1979). In this case, we have an interval scale. Quaddus (1987) calls $\alpha_{i}$ and $\beta_{i}$ "costs," which might suggest measuring them in dollar amounts and therefore possibly on a ratio scale. However, he points out that techniques from preference and value theory, such as those developed by Keeney and Raiffa (1976), can be used to estimate these costs; this suggests that they are measures of utility or value. Hall and Posner (1991) speak of the $\alpha_{i}$ and $\beta_{i}$ as weights or "values," which suggests again that they could be measured on scales other than ratio scales. Many authors, for example Elsayed et al. (1993), refer to $\alpha_{i}$ and $\beta_{i}$ as penalties arising in connection with "just-in-time" production problems. Penalties in such problems are often measured in terms of "customer satisfaction" (see for example Auguston 1989), which certainly could be measured on any of a number of different types of scales.

As the above discussion shows, the conclusion that $S$ is an optimal schedule may not be meaningful if weights are measured on interval scales. In the next section, we systematically study the four objective functions defined above if weights are measured on interval scales or ordinal scales and determine the meaningfulness of the conclusion of optimality under the sixteen cases defined by the presence or absence of the conditions $\mathrm{cw}$, cdd, cpt, and s.

\section{MEANINGFULNESS OF THE CONCLUSION OF OPTIMALITY: THE CASE OF INTERVAL AND ORDINAL SCALES}

Our results are summarized in Table I. They show that with interval or ordinal scales, and common and symmetric weights, the conclusion of optimality is meaningful for all the objective functions we have considered, but with common and nonsymmetric weights, it can be meaningless for $F_{\text {sum } E \mid T}$ and $F_{\max E \mid T}$. With noncommon weights, the conclusion of optimality can be meaningless in all cases except the ncw, cdd, cpt, s case for $F_{\text {sum } E \mid T}$ and the corresponding ncw, cdd, cpt, ns case for $F_{\text {sum } T}$. The detailed conclusions are not as important as the principle that the conclusion of optimality may indeed be meaningless and that it is necessary to take considerations of meaningfulness into account in analysis of scheduling problems and, more generally, of problems of operations research.

The entries in Table I are justified by a series of examples, theorems, and principles that give us a way of constructing new examples from old ones. Some of the details are left to the appendix at the end. The reader who is most interested in the general principles developed in this paper or who wishes to use the table for reference without going 
Table I

Meaningfulness of the Conclusion of Optimality Under Either Interval or Ordinal Scales

\begin{tabular}{|c|c|c|c|c|}
\hline Case & $F_{\text {sumE } \mid T}$ & $F_{\text {sum } T}$ & $F_{\max \mid T}$ & $F_{\max T}$ \\
\hline $\mathrm{cw}$ cdd $\mathrm{cpt} \mathrm{s}$ & $\begin{array}{l}\text { Yes } \\
\text { T } 2.1\end{array}$ & ND & $\begin{array}{l}\text { Yes } \\
\text { T } 2.3\end{array}$ & ND \\
\hline cw cdd cpt ns & $\begin{array}{l}\text { No } \\
\text { E1 }\end{array}$ & $\begin{array}{l}\text { Yes } \\
\text { T } 2.2\end{array}$ & $\begin{array}{l}\text { No } \\
\text { E8 }\end{array}$ & $\begin{array}{l}\text { Yes } \\
\text { T } 2.4\end{array}$ \\
\hline cw cdd ncpt s & $\begin{array}{l}\text { Yes } \\
\text { T } 2.1\end{array}$ & ND & $\begin{array}{l}\text { Yes } \\
\text { T } 2.3\end{array}$ & ND \\
\hline cw cdd ncpt ns & $\begin{array}{l}\text { No } \\
\text { E2 }\end{array}$ & $\begin{array}{l}\text { Yes } \\
\text { T } 2.2\end{array}$ & $\begin{array}{l}\text { No } \\
\text { E9 }\end{array}$ & $\begin{array}{l}\text { Yes } \\
\text { T } 2.4\end{array}$ \\
\hline cw ncdd cpt $s$ & $\begin{array}{l}\text { Yes } \\
\text { T } 2.1\end{array}$ & ND & $\begin{array}{l}\text { Yes } \\
\text { T } 2.3\end{array}$ & ND \\
\hline cw ncdd cpt ns & $\begin{array}{c}\text { No } \\
\mathrm{E} 1+\mathrm{P} 1\end{array}$ & $\begin{array}{l}\text { Yes } \\
\text { T } 2.2\end{array}$ & $\begin{array}{c}\text { No } \\
\mathrm{E} 8+\mathrm{P} 1\end{array}$ & $\begin{array}{l}\text { Yes } \\
\text { T } 2.4\end{array}$ \\
\hline cw ncdd ncpt $s$ & $\begin{array}{l}\text { Yes } \\
\text { T } 2.1\end{array}$ & ND & $\begin{array}{l}\text { Yes } \\
\text { T } 2.3\end{array}$ & ND \\
\hline cw ncdd ncpt ns & $\begin{array}{c}\text { No } \\
\mathrm{E} 1+\mathrm{P} 1\end{array}$ & $\begin{array}{c}\text { Yes } \\
\text { T } 2.2\end{array}$ & $\begin{array}{c}\text { No } \\
\mathrm{E} 8+\mathrm{P} 1\end{array}$ & $\begin{array}{l}\text { Yes } \\
\text { T } 2.4\end{array}$ \\
\hline ncw cdd cpt $\mathrm{s}$ & $\begin{array}{l}\text { Yes } \\
\text { T } 2.5\end{array}$ & ND & $\begin{array}{l}\text { No } \\
\text { E10 }\end{array}$ & ND \\
\hline ncw cdd cpt ns & $\begin{array}{l}\text { No } \\
\text { E3 }\end{array}$ & $\begin{array}{l}\text { Yes } \\
\text { T } 2.6\end{array}$ & $\begin{array}{c}\text { No } \\
\mathrm{E} 8+\mathrm{P} 2\end{array}$ & $\stackrel{*}{\mathrm{~T} 2.7+\mathrm{E} 14}$ \\
\hline ncw cdd ncpt $s$ & $\begin{array}{l}\text { No } \\
\text { E4 }\end{array}$ & ND & $\begin{array}{l}\text { No } \\
\text { E11 }\end{array}$ & ND \\
\hline ncw cdd ncpt ns & $\begin{array}{l}\text { No } \\
\text { E3 }\end{array}$ & $\begin{array}{l}\text { No } \\
\text { E6 }\end{array}$ & $\begin{array}{c}\text { No } \\
\mathrm{E} 8+\mathrm{P} 2\end{array}$ & T $2.7+\mathrm{E} 15$ \\
\hline ncw ncdd $\mathrm{cpt} s$ & $\begin{array}{l}\text { No } \\
\text { E5 }\end{array}$ & ND & $\begin{array}{c}\text { No } \\
\mathrm{E} 10+\mathrm{P} 1\end{array}$ & ND \\
\hline ncw nodd cpt ns & $\begin{array}{c}\text { No } \\
\mathrm{E} 1+\mathrm{P} 1\end{array}$ & $\begin{array}{l}\text { No } \\
\text { E7 }\end{array}$ & $\begin{array}{c}\text { No } \\
\mathrm{E} 8+\mathrm{P} 1\end{array}$ & $\begin{array}{l}\text { No } \\
\text { E12 }\end{array}$ \\
\hline ncw ncdd ncpt s & $\begin{array}{c}\text { No } \\
\mathrm{E} 4+\mathrm{P} 1\end{array}$ & ND & $\begin{array}{c}\text { No } \\
\mathrm{E} 10+\mathrm{P} 1\end{array}$ & ND \\
\hline ncw ncdd ncpt ns & $\begin{array}{c}\text { No } \\
\mathrm{E} 1+\mathrm{P} 1\end{array}$ & $\begin{array}{c}\text { No } \\
\text { E6 + P1 }\end{array}$ & $\begin{array}{c}\text { No } \\
\mathrm{E} 8+\mathrm{P} 1\end{array}$ & $\begin{array}{l}\text { No } \\
\text { E13 }\end{array}$ \\
\hline
\end{tabular}

Yes = meaningful, No = can be meaningless (see Remark after Example 1),

$\mathrm{ND}=$ case not defined,

$\mathrm{Ei}=$ justified by Example $\mathrm{i}, \mathrm{Ti}=$ justified by Theorem $\mathrm{i}$,

$\mathrm{Ei}+\mathrm{Pj}=$ justified by example constructed from Example $\mathrm{i}$ by Principle $\mathrm{j}$,

$\mathrm{cw}=$ common weights, $\mathrm{ncw}=$ noncommon weights,

cdd $=$ common due dates, ncdd $=$ noncommon due dates,

$\mathrm{cpt}=\mathrm{common}$ processing times, $\mathrm{ncpt}=$ noncommon processing times,

$\mathrm{s}=$ symmetric, $\mathrm{ns}=$ nonsymmetric (by notational convention, ns applies to $F_{\operatorname{sum} T}$ and $F_{\max T}$ ).

* = meaningful with LWO schedule but not necessarily with other optimal schedules. See remark following Theorem 2.7

through the detailed justifications may wish to skip the appendix. Since one example suffices to show that a conclusion can be meaningless, the reader might naturally ask if the examples we present are simply isolated cases. The answer is that they are not, and that it is not hard to produce many other examples. It should also be noted that, in our examples, $d_{i}, p_{i}, \alpha_{i}, \beta_{i}$ are rational numbers. Hence, it is straightforward to modify our examples to produce new examples with integer processing times and/or integer desired completion times and/or integer weights.
Throughout this section, we adopt the convention that when scale values are required to be positive, by admissible transformations of interval scales we mean the transformations $x$ into $k x+l, k>0$, that leave all weights positive; in other words, $k>0$ and $l$ must be chosen so that all $k \alpha_{i}+l$ and $k \beta_{i}+l$ are positive. We still call the corresponding scale an interval scale although technically not all transformations of the form $x$ into $k x+l$ are allowed. Similarly, if scale values are required to be positive by admissible transformations of ordinal scales we mean the (strictly) monotone increasing 
transformations that take positive real numbers into positive real numbers.

Example 1. Suppose that the weights $\alpha_{i}, \beta_{i}, i=1, \ldots, n$, are measured on an interval or ordinal scale. If the objective function is $F_{\text {sumE } \mid T}$, then in the cw, cdd, cpt, ns case, the conclusion of optimality can be meaningless.

Remark. Before justifying Example 1, we explain what we mean in this paper by the conclusion "can be meaningless" in a given case. By this, we mean that there are instances of scheduling problems where weights are measured on an interval or ordinal scale and the assumptions of the case hold, but where no schedule is optimal for all acceptable choices of scale. To verify this, it suffices to give examples of scheduling problems and schedules $S^{\prime}$ so that $S^{\prime}$ is not optimal under one choice of scale of measurement, but $S^{\prime}$ is optimal after an admissible transformation of scale under which no previously optimal schedule remains optimal. When we say that a particular case is meaningful, we mean that the conclusion of optimality of $S$ is meaningful for any optimal $S$. We will conclude that in two cases, there is always an optimal schedule $S$ for which the conclusion of optimality is meaningful, but there might be other optimal schedules for which this conclusion is not meaningful.

Remark. Note that every transformation $x$ into $k x+l$, $k>0$, that leaves all weights positive (i.e., an admissible transformation of weights that are measured on an interval scale) is a (strictly) monotone increasing transformation of positive real numbers (i.e., it can be viewed as an admissible transformation of weights that are measured on an ordinal scale). Hence, in order to show that the conclusion of optimality can be meaningless if the weights are measured on an ordinal scale, it suffices to show that the conclusion of optimality can be meaningless if the weights are measured on an interval scale. Conversely, if the conclusion of optimality is meaningful when weights are measured on an ordinal scale, then the conclusion of optimality is meaningful when weights are measured on an interval scale.

Proof of Example 1. The example here is the example given in the previous section, and we simply give it a number here so we may refer to it in Table I. To summarize, we have $n=3, d=3, p=1, \alpha=1, \beta=10$. The schedule $S$ defined by $C(S)=(1,2,3)$ is the unique optimal schedule (up to symmetry), but if $k=1, l=100$, then with $C\left(S^{\prime}\right)=(2,3,4)$, we have after transformation of weights that $F_{\text {sum } E \mid T}^{\prime}\left(S^{\prime}\right)<F_{\text {sum } E \mid T}^{\prime}(S)$. So $S$ is no longer optimal.

Remark. We say in Example 1 (and Table I) that the condition of optimality can be meaningless. As we have noted, this is simply to say that there are examples satisfying the case $\mathrm{cw}$, cdd, cpt, ns under the objective function
$F_{\text {sumE|T }}$ for which no schedule is optimal for all acceptable choices of scale. It is not to say that all situations satisfying these conditions under this objective function will make the conclusion meaningless. For instance, let us take $n=$ $2, d=2, p=1, \alpha=1, \beta=10$, and let $C(S)=(1,2)$. It can be seen that $S$ is optimal and that it remains optimal after a transformation $x$ into $k x+l$ with $k>0$ and $\alpha^{\prime}=$ $k \alpha+l>0$ and $\beta^{\prime}=k \beta+l>0$. Actually, it suffices to prove the latter because a special case has $k=1, l=0$, which gives us back $\alpha$ and $\beta$.

Example 2. Suppose that the weights $\alpha_{i}, \beta_{i}, i=1, \ldots, n$, are measured on an interval or ordinal scale. If the objective function is $F_{\text {sumE|T}}$, then in the cw, cdd, ncpt, ns case, the conclusion of optimality can be meaningless.

Proof. Let $n=3, d=3.1, p=(1,1,1.1), \alpha=1, \beta=10$. Then $C(S)=(2.1,3.1,1.1)$ is the unique optimal schedule up to symmetry among the first two jobs (see appendix). Now, let $k=1, l=100$. Then after change of scale, we get $\alpha^{\prime}=101, \beta^{\prime}=110$. We have

$$
\begin{aligned}
& F_{\text {sumE } \mid T}^{\prime}(S)=101(3.1-2.1)+110(3.1-3.1) \\
& \quad+101(3.1-1.1)=303 .
\end{aligned}
$$

However, if $C\left(S^{\prime}\right)=(2,3,4.1)$, we have

$$
\begin{aligned}
& F_{\text {sumE } \mid T}^{\prime}\left(S^{\prime}\right)=101(3.1-2)+101(3.1-3) \\
& \quad+110(4.1-3.1)=231.2,
\end{aligned}
$$

and so $S$ is no longer optimal.

Remark. Example 2 can be generalized to give one example that covers all cases with cw, ns. We take $n=3, d=$ $(3,3,3+q), p=(1,1,1+r), \alpha=1, \beta=10$, with $q, r \geqslant$ $0,|q-r|<1$. If $C(S)=(1+x, 2+x, 3+q)$, where $x=$ $q-r$, then $S$ turns out to be the optimal schedule up to symmetry. However, if $k=1, l=100$, then after the corresponding change of scale, $S^{\prime}$ with $C\left(S^{\prime}\right)=(2,3,4+$ $r$ ) has a lower objective function value than $S$. Details are a bit messy and are left to the reader.

Example 3. Suppose that the weights $\alpha_{i}, \beta_{i}, i=1, \ldots, n$, are measured on an interval or ordinal scale. If the objective function is $F_{\text {sumE|T}}$, then in the ncw, cdd, cpt, ns and ncw, cdd, ncpt, ns cases, the conclusion of optimality can be meaningless.

Proof. Let $n=3, d=2, p=(1,1,1+q)$ with $q=0$ or $.01, \boldsymbol{\alpha}=\left(\frac{1}{2}, \frac{1}{3}, 1\right), \boldsymbol{\beta}=\left(\frac{1}{16}, \frac{1}{81}, 1\right)$, and take $C(S)=(3,4,2)$. The case $q=0$ of course will give us cpt, while the case $q=.01$ will give us ncpt. Then $S$ is the unique optimal schedule (see appendix). Let $k=1, l=1 / 2$, giving us $\boldsymbol{\alpha}^{\prime}=$ $\left(1, \frac{5}{6}, \frac{3}{2}\right), \boldsymbol{\beta}^{\prime}=\left(\frac{9}{16}, \frac{83}{162}, \frac{3}{2}\right)$. Now we have

$$
\begin{aligned}
F_{\text {sumE|T}}^{\prime}(S)= & \frac{9}{16}(3-2)+\frac{83}{162}(4-2) \\
& +\frac{3}{2}(2-2)>1.58 .
\end{aligned}
$$

If $C\left(S^{\prime}\right)=(1,3+q, 2+q)$, then 


$$
\begin{aligned}
F_{\text {sumE|T }}^{\prime}\left(S^{\prime}\right)= & 1 \cdot(2-1)+\frac{83}{162}(3+q-2) \\
& +\frac{3}{2}(2+q-2) \\
= & 1+\frac{83}{162}+q\left(\frac{83}{162}+\frac{3}{2}\right)<1.55,
\end{aligned}
$$

since $q \leqslant 0.01$. We conclude that $S$ is no longer optimal.

Example 4. Suppose that the weights $\alpha_{i}, \beta_{i}, i=1, \ldots, n$, are measured on an interval or ordinal scale. If the objective function is $F_{\text {sum } E \mid T}$, then in the ncw, cdd, ncpt, s case, the conclusion of optimality can be meaningless.

Proof. Let $n=3, d=0, \boldsymbol{p}=(1,1,2), \boldsymbol{\alpha}=\boldsymbol{\beta}=(2,2,5)$. Let $C(S)=(3,4,2)$. Then $S$ is optimal. (Note that, by symmetry, $(4,3,2)$ defines the only other optimal schedule). To see why, note that since $d_{i}=0$ for all $i$, an optimal schedule $S=\left(C_{1}, C_{2}, C_{3}\right)$ will start at time 0 , have no idle times, and $C_{i} \geqslant C_{j} \Leftrightarrow C_{i} / \beta_{i} \geqslant C_{j} / \beta_{j}, i, j=1,2,3$ (as proved by Smith 1956). (In the present example, this is simple to show. In the case where job 1 completes before job 2 , the only other candidates for optimal schedule are $(1,4,3)$ and $(1,2,4)$, and it is easy to show that $F_{\text {sumE|T }}$ is larger for these two schedules than for $S$.) Now take $k=1$, $l=3$, giving $\alpha^{\prime}=\beta^{\prime}=(5,5,8)$. We have $F_{\text {sum } E \mid T}^{\prime}(S)=51$, but $F_{\text {sumE|T}}^{\prime}\left(S^{\prime}\right)=49$ for schedule $S^{\prime}$ defined by $C\left(S^{\prime}\right)=$ $(1,4,3)$. So $S$ is no longer optimal.

Remark. We have used $d=0$ in the previous example. If that makes the reader uncomfortable, we could use an arbitrary $d$, add a fourth job with $p_{4}=d, \alpha_{4}=\beta_{4}=10^{10}$. Then it is clear that the fourth job is always first and the rest of the argument is as in Example 4.

Example 5. Suppose that the weights $\alpha_{i}, \beta_{i}, i=1, \ldots, n$, are measured on an interval or ordinal scale. If the objective function is $F_{\text {sumE|T }}$, then in the ncw, ncdd, cpt, s case, the conclusion of optimality can be meaningless.

Proof. Let $n=4, \boldsymbol{d}=(2,2,2,1), p=1, \boldsymbol{\alpha}=\boldsymbol{\beta}=(9,9,9$, 1). Then $C(S)=(2,1,3,4)$ is the unique optimal schedule up to symmetry among first three jobs (see appendix). Now, let $k=\frac{1}{8}, l=\frac{7}{8}$. Then $\boldsymbol{\alpha}=\boldsymbol{\beta}^{\prime}=(2,2,2,1)$ and

$$
\begin{aligned}
F_{\text {sumE } \mid T}^{\prime}(S)= & 2(2-2)+2(2-1)+2(3-2) \\
& +1 \cdot(4-1)=7 .
\end{aligned}
$$

However, if $C\left(S^{\prime}\right)=(2,3,4,1)$, then

$$
\begin{aligned}
F_{\text {sumE|T }}^{\prime}\left(S^{\prime}\right)= & 2(2-2)+2(3-2)+2(4-2) \\
& +1 \cdot(1-1)=6,
\end{aligned}
$$

so $S$ is no longer optimal.

Example 6. Suppose that the weights $\beta_{i}, i=1, \ldots, n$, are measured on an interval or ordinal scale. If the objective function is $F_{\text {sum } T}$, then in the ncw, cdd, ncpt, ns case, the conclusion of optimality can be meaningless.
Proof. Let $n=2, d=1, p=(1,2), \boldsymbol{\beta}=(1,3)$. Let $C(S)$ $=(3,2)$. Since only tardiness enters into the objective function and $p_{i} \geqslant 1=d$, an optimal schedule will start at time 0 and have no idle time. Thus, we need only consider the alternative schedule $S^{\prime}$ given by $C\left(S^{\prime}\right)=(1,3)$. We have $F_{\text {sum } T}(S)=5$, while $F_{\text {sum } T}\left(S^{\prime}\right)=6$, so $S$ is optimal. However, if we take $k=1, l=2$, we get $\boldsymbol{\beta}^{\prime}=(3,5)$ and now $F_{\text {sum } T}^{\prime}(S)=11$ while $F_{\text {sum } T}^{\prime}\left(S^{\prime}\right)=10$. So $S$ is no longer optimal.

Example 7. Suppose that the weights $\beta_{i}, i=1, \ldots, n$, are measured on an interval or ordinal scale. If the objective function is $F_{\text {sum } T}$, then in the ncw, ncdd, cpt, ns case, the conclusion of optimality can be meaningless.

Proof. Let $n=4, \boldsymbol{d}=(2,2,2,1), p=1, \boldsymbol{\beta}=(9,9,9,1)$. Then $C(S)=(1,2,3,4)$ is the unique optimal schedule up to symmetry among first three jobs (see appendix). Now let $k=\frac{5}{8}, l=\frac{99}{8}$. Then $\boldsymbol{\beta}^{\prime}=(18,18,18,13)$ and

$F_{\text {sum } T}^{\prime}(S)=18 \cdot 0+18 \cdot 1+13 \cdot 3=57$,

while if $C\left(S^{\prime}\right)=(2,3,4,1)$,

$F_{\text {sum } T}^{\prime}\left(S^{\prime}\right)=18 \cdot 0+18 \cdot 1+18 \cdot 2+13 \cdot 0=54$.

Hence, $S$ is no longer optimal.

Example 8. Suppose that the weights $\alpha_{i}, \beta_{i}, i=1, \ldots, n$, are measured on an interval or ordinal scale. If the objective function is $F_{\max E \mid T}$, then in the cw, cdd, cpt, ns case, the conclusion of optimality can be meaningless.

Proof. Let $n=3, d=3, p=1, \alpha=1, \beta=10$. Then $C(S)=\left(\frac{13}{11}, \frac{24}{11}, \frac{35}{11}\right)$ is the unique optimal schedule up to symmetry among all three jobs (see appendix). Now take $k=1, l=100$, giving us $\alpha^{\prime}=101, \beta^{\prime}=110$. It follows that

$F_{\max \mid T}^{\prime}(S) \geqslant 101\left(3-\frac{13}{11}\right)>180$.

However, if $C\left(S^{\prime}\right)=(2,3,4)$, then

$$
\begin{aligned}
& F_{\max E \mid T}^{\prime}\left(S^{\prime}\right)=\max \{101(3-2), 110(3-3), \\
& 110(4-3)\}=110<180,
\end{aligned}
$$

and we conclude that $S$ is no longer optimal.

Example 9. Suppose that the weights $\alpha_{i}, \beta_{i}, i=1, \ldots, n$, are measured on an interval or ordinal scale. If the objective function is $F_{\max E \mid T}$, then in the cw, cdd, ncpt, ns case, the conclusion of optimality can be meaningless.

Proof. Let $n=3, d=3, p=(1,1,1.1), \alpha=1, \beta=10$. Then $C(S)=\left(\frac{24}{11}, \frac{35}{11}, \frac{13}{11}\right)$ is the unique optimal schedule up to symmetry among first two jobs (see appendix). Now, let $k=1, l=100$, giving us $\alpha^{\prime}=101, \beta^{\prime}=110$. It follows that

$F_{\max E \mid T}^{\prime}(S) \geqslant 101\left(3-\frac{13}{11}\right)>180$.

However, if $C\left(S^{\prime}\right)=(2,3,4.1)$, then 
$F_{\max E \mid T}^{\prime}\left(S^{\prime}\right)=\max \{101(3-2), 110(3-3)$,

$110(4.1-3)\}=121$.

Hence, $S$ is no longer optimal.

Example 10. Suppose that the weights $\alpha_{i}, \beta_{i}, i=1, \ldots, n$, are measured on an interval or ordinal scale. If the objective function is $F_{\max E \mid T}$, then in the ncw, cdd, cpt, s case, the conclusion of optimality can be meaningless.

Proof. Let $n=2, d=2, p=1, \boldsymbol{\alpha}=\boldsymbol{\beta}=(1,2)$. Then the only optimal schedules are $C(S)=\left(\frac{8}{3}, \frac{5}{3}\right)$ and $C\left(S^{\prime}\right)=\left(\frac{4}{3}, \frac{7}{3}\right)$ (see appendix). Let $k=1$ and $l=1$, giving us $\boldsymbol{\alpha}^{\prime}=\boldsymbol{\beta}^{\prime}=$ $(2,3)$. Then

$F_{\max E \mid T}^{\prime}(S)=\max \left\{2\left(\frac{8}{3}-2\right), 3\left(2-\frac{5}{3}\right)\right\}=\frac{4}{3}$,

$F_{\max \mid T}^{\prime}\left(S^{\prime}\right)=\max \left\{2\left(2-\frac{4}{3}\right), 3\left(\frac{7}{3}-2\right)\right\}=\frac{4}{3}$,

while if $C\left(S^{\prime \prime}\right)=\left(\frac{7}{5}, \frac{12}{5}\right)$, we have

$F_{\max E \mid T}^{\prime}\left(S^{\prime \prime}\right)=\max \left\{2\left(2-\frac{7}{5}\right), 3\left(\frac{12}{5}-2\right)\right\}=\frac{6}{5}$.

We conclude that both $S$ and $S^{\prime}$ are no longer optimal.

Example 11. Suppose that the weights $\alpha_{i}, \beta_{i}, i=1, \ldots, n$, are measured on an interval or ordinal scale. If the objective function is $F_{\max E \mid T}$, then in the ncw, cdd, ncpt, s case, the conclusion of optimality can be meaningless.

Proof. Let $n=2, d=3, p=(1,2), \alpha=\beta=(1,2)$. Then $C(S)=\left(\frac{11}{3}, \frac{8}{3}\right)$ is the unique optimal schedule (see appendix). Let $k=1, l=1$, giving us $\boldsymbol{\alpha}^{\prime}=\boldsymbol{\beta}^{\prime}=(2,3)$. Then

$F_{\max E \mid T}^{\prime}(S)=\max \left\{2\left(\frac{11}{3}-3\right), 3\left(3-\frac{8}{3}\right)\right\}=\frac{4}{3}$,

while if $C\left(S^{\prime}\right)=\left(\frac{18}{5}, \frac{13}{5}\right)$, we have

$F_{\max \mid T}^{\prime}\left(S^{\prime}\right)=\max \left\{2\left(\frac{18}{5}-3\right), 3\left(3-\frac{13}{5}\right)\right\}=\frac{6}{5}$.

We conclude that $S$ is no longer optimal.

Example 12. Suppose that the weights $\beta_{i}, i=1, \ldots, n$, are measured on an interval or ordinal scale. If the objective function is $F_{\max T}$, then in the ncw, ncdd, cpt, ns case, the conclusion of optimality can be meaningless.

Proof. Let $n=2, p=2, d=(1,3), \boldsymbol{\beta}=(1,4)$. Note that an optimal schedule will start at time 0 and have no idle time (since only tardiness enters into the objective function). Thus, we only need to consider two schedules $C(S)$ $=(4,2)$ and $C\left(S^{\prime}\right)=(2,4)$. Hence, $S$ is the unique optimal schedule (because $F_{\max T}(S)=3<4=$ $\left.F_{\max T}\left(S^{\prime}\right)\right)$. Let $k=1, l=1$, giving us $\boldsymbol{\beta}^{\prime}=(2,5)$. Then $F_{\max T}^{\prime}(S)=6>5=F_{\max T}^{\prime}\left(S^{\prime}\right)$ and we conclude that $S$ is no longer optimal.

Example 13. Suppose that the weights $\beta_{i}, i=1, \ldots, n$, are measured on an interval or ordinal scale. If the objec- tive function is $F_{\max }$, then in the ncw, ncdd, ncpt, ns case, the conclusion of optimality can be meaningless.

Proof. Let $n=2, p=(2,3), d=(1,4), \boldsymbol{\beta}=(1,5)$. As in Example 12 we note that an optimal schedule will start at time 0 and have no idle time. Thus, we only need to consider two schedules $C(S)=(5,3)$ and $C\left(S^{\prime}\right)=(2,5)$. Hence, $S$ is the unique optimal schedule (because $F_{\max T}(S)$ $\left.=4<5=F_{\max T}\left(S^{\prime}\right)\right)$. Let $k=1, l=1$, giving us $\boldsymbol{\beta}^{\prime}=(2$, $6)$. Then $F_{\max T}^{\prime}(S)=8>6=F_{\max T}^{\prime}\left(S^{\prime}\right)$ and we conclude that $S$ is no longer optimal.

We now present some positive results. The following theorems show for which of the scheduling problems we have considered the conclusion of optimality is meaningful if the weights are measured on an interval or ordinal scale.

Theorem 2.1. Suppose that the weights $\alpha_{i}, \beta_{i}, i=1, \ldots, n$, are measured on an interval or ordinal scale. If the objective function is $F_{\mathrm{sum} E \mid T}$, then in all cases in which $\mathrm{cw}, \mathrm{s}$ hold, the conclusion of optimality is meaningful.

Proof. We have

$F_{\mathrm{sum} E \mid T}\left(S^{\prime}\right)=\alpha \sum_{i=1}^{n} E_{i}\left(S^{\prime}\right)+\alpha \sum_{i=1}^{n} T_{i}\left(S^{\prime}\right)$.

Thus, for an admissible transformation of scale $\Phi$,

$$
\begin{aligned}
F_{\text {sum } E \mid T}^{\prime}\left(S^{\prime}\right) & =\Phi(\alpha) \sum_{i=1}^{n} E_{i}\left(S^{\prime}\right)+\Phi(\alpha) \sum_{i=1}^{n} T_{i}\left(S^{\prime}\right) \\
& =\frac{\Phi(\alpha)}{\alpha} F_{\text {sum } E \mid T}\left(S^{\prime}\right) .
\end{aligned}
$$

By our convention, $\alpha>0$ and $\Phi(\alpha)>0$. Thus,

$F_{\text {sumE } \mid T}(S) \leqslant F_{\text {sumE } \mid T}\left(S^{\prime}\right)$ if and only if

$\frac{\Phi(\alpha)}{\alpha} F_{\text {sum } E \mid T}(S) \leqslant \frac{\Phi(\alpha)}{\alpha} F_{\text {sumE|T }}\left(S^{\prime}\right)$ if and only if

$F_{\text {sum } E \mid T}^{\prime}(S) \leqslant F_{\text {sum } E \mid T}^{\prime}\left(S^{\prime}\right)$.

The next three theorems can also be proved similarly.

Theorem 2.2. Suppose that the weights $\beta_{i}, i=1, \ldots, n$, are measured on an interval or ordinal scale. If the objective function is $F_{\mathrm{sum} T}$, then in all cases in which $\mathrm{cw}$ holds, the conclusion of optimality is meaningful.

Theorem 2.3. Suppose that the weights $\alpha_{i}, \boldsymbol{\beta}_{i}, i=1, \ldots, n$, are measured on an interval or ordinal scale. If the objective function is $F_{\max E \mid T}$, then in all cases in which $\mathrm{cw}, \mathrm{s}$ hold, the conclusion of optimality is meaningful.

Theorem 2.4. Suppose that the weights $\beta_{i}, i=1, \ldots, n$, are measured on an interval or ordinal scale. If the objective function is $F_{\max T}$, then in all cases in which $\mathrm{cw}$ holds, the conclusion of optimality is meaningful.

Theorem 2.5. Suppose that the weights $\alpha_{i}, \beta_{i}, i=1, \ldots, n$, are measured on an interval or ordinal scale. If the objective function is $F_{\mathrm{sum} E \mid T}$, then in all cases in which cdd, $c p t$, $s$ hold, the conclusion of optimality is meaningful. 
Proof. Suppose without loss of generality that

$\alpha_{1} \geqslant \cdots \geqslant \alpha_{n}$.

We consider a greedy schedule (see Kanet 1981, Sundararaghavan and Ahmed 1984, Hall 1986). Let $j$ be the largest integer such that $d-j p \geqslant p$. Then one greedy schedule is schedule $T$ defined by

$$
\begin{aligned}
& C(T)=(d, d-p, d+p, d-2 p, d+2 p, \ldots, \\
& \quad d-j p, d+j p, d+(j+1) p, d+(j+2) p, \ldots) .
\end{aligned}
$$

Note that we cannot use $d-(j+1) p$ because $C_{i} \geqslant p$. All other greedy schedules are obtained from $T$ by switching $C_{2 i}$ and $C_{2 i+1}$ for possibly some $i$ with $C_{2 i} \leqslant d-j p$, and by switching $C_{i}$ and $C_{j}$ for possibly some pairs $(i, j)$ with $\alpha_{i}=$ $\alpha_{j}$.

We say that $(i, j)$ is a reversing pair for $S$ if $\alpha_{i}>\alpha_{j}$ and $\left|C_{i}-d\right|>\left|C_{j}-d\right|$. It is easy to see that $S$ with reversing pairs cannot be optimal: simply switch $C_{i}$ and $C_{j}$ to obtain a schedule $S^{\prime}$ with a smaller $F_{\text {sumE|T }}\left(S^{\prime}\right)$. Also, since no two $C_{i}$ can be equal, at most two $\left|C_{i}-d\right|$ can be equal. It follows that $S$ is optimal if and only if it is greedy.

We complete the proof by noting that any (strictly) monotone increasing transformation of positive real numbers doesn't change the greedy schedules since the ordering of weights is unchanged. Thus, the same schedules remain optimal.

Theorem 2.6. Suppose that the weights $\beta_{i}, i=1, \ldots, n$, are measured on an interval or ordinal scale. If the objective function is $F_{\text {sum } T}$, then in all cases in which cdd, cpt hold, the conclusion of optimality is meaningful.

Proof. The proof is similar to that of Theorem 2.5. We suppose without loss of generality that

$\beta_{1} \geqslant \cdots \geqslant \beta_{n}$.

Then the greedy schedules are those obtained from

$C(T)=(0, p, 2 p, \ldots)$,

by switching some $C_{i}$ and $C_{j}$ that are less than or equal to $d$ (since we do not penalize earliness) and switching $C_{i}$ and $C_{j}$ for possibly some pairs $(i, j)$ with $\beta_{i}=\beta_{j}$. It is easy to see that $S$ is optimal if and only if it is greedy and that any (strictly) monotone increasing transformation of positive real numbers does not change the greedy schedule because it does not change the order of weights.

In all scheduling problems that we have considered so far, we have concluded that either the conclusion of optimality is meaningful for any optimal schedule (when weights are measured on an ordinal scale) or we have given an example where the conclusion of optimality is meaningless for all optimal schedules (when the weights are measured on an interval or ordinal scale). If the objective function is $F_{\max T}$ and if cdd holds, we can only conclude that there exists an optimal schedule $S$ such that the conclusion of optimality is always meaningful.
Theorem 2.7. Suppose that the weights $\beta_{i}, i=1, \ldots, n$, are measured on an interval or ordinal scale. If the objective function is $F_{\max }$, then in all cases in which cdd holds, there exists an optimal schedule $S$ for which the conclusion of optimality is meaningful.

Proof. We first note that any optimal schedule will start at time 0 and have no idle time (this is because only tardiness enters into the objective function). Any such schedule is completely determined by an ordering of jobs. Let $S$ be a schedule starting at time 0 with no idle times such that $\beta_{i}>\beta_{j} \Rightarrow C_{i}<C_{j}, i, j=1, \ldots, n$. In other words, $S$ is defined by the largest weight order (LWO), that is an ordering of jobs in which a job with the larger weight precedes a job with the smaller weight. We will also refer to $S$ as an LWO schedule.

It is straightforward to see that $S$ is optimal. To see why note that in any other schedule $S^{\prime}$ (that is not given by an LWO), there exist jobs $i$ and $j$ such that $C_{j}^{\prime}=C_{i}^{\prime}+p_{j}(j$ is scheduled immediately after $i$ ) and such that $\beta_{j}>\beta_{i}$. Then, for schedule $S^{\prime \prime}$ obtained from $S^{\prime}$ just by switching the ordering of the two consecutive jobs $i$ and $j$ (i.e., $S^{\prime}$ schedules $j$ before $i$ ), we have: $C_{i}^{\prime \prime}=C_{j}^{\prime}, C_{j}^{\prime \prime}=C_{i}^{\prime}-p_{i}+p_{j}$, and $C_{k}^{\prime \prime}=C_{k}^{\prime}$ for any $k \neq i, j$. Since cdd holds, we also have $T_{i}^{\prime \prime}=T_{j}^{\prime}, T_{j}^{\prime \prime} \leqslant T_{j}^{\prime}$, and $T_{k}^{\prime \prime}=T_{k}^{\prime}, k \neq i, j$. Note that

$\max \left\{\beta_{j} T_{j}^{\prime}, \beta_{i} T_{i}^{\prime}\right\}=\beta_{j} T_{j}^{\prime} \geqslant \max \left\{\beta_{j} T_{j}^{\prime \prime}, \beta_{i} T_{i}^{\prime \prime}\right\}$,

and we conclude that $F_{\max T}\left(S^{\prime}\right) \geqslant F_{\max T}\left(S^{\prime \prime}\right)$ (since $\beta_{k} T_{k}^{\prime \prime}=$ $\beta_{k} T_{k}^{\prime}$ for any $\left.k \neq i, j\right)$. Hence, by induction, we conclude that there exists an optimal schedule $S^{*}$ given by an LWO. Clearly, for any two LWO schedules, $S$ and $S^{*}$, we have $F_{\max T}(S) \geqslant F_{\max T}\left(S^{*}\right)$. Therefore, $S$ is optimal.

Finally, note that any (strictly) monotone increasing transformation of positive real numbers does not change the largest weight order. Hence, for an optimal schedule $S$ given by a largest weight order, the conclusion of optimality is meaningful.

Remark. Although the conclusion of optimality is meaningful for any schedule given by the largest weight order, it is possible that there exist other optimal schedules for which the conclusion of optimality is meaningless (even if the weights are measured on an interval scale). The following two examples illustrate this.

Example 14. Suppose that the weights $\beta_{i}, i=1, \ldots, n$, are measured on an interval or ordinal scale. If the objective function is $F_{\max T}$, then in the ncw, cdd, cpt, ns case, there are instances of the scheduling problem and schedules for which the conclusion of optimality is meaningless.

Proof. Let $n=4, p=1, d=1$, and $\beta=(10,9,3,2)$. Then $C\left(S^{*}\right)=(1,2,4,3)$ defines an optimal schedule which is not an LWO schedule. (It is straightforward to check that $S^{*}$ is optimal since, for any schedule $S^{\prime}$, either $C_{1}^{\prime} \geqslant 2$ or $C_{2}^{\prime} \geqslant 2$, i.e., $T_{1}^{\prime}=C_{1}^{\prime}-1 \geqslant 1$ or $T_{2}^{\prime}=C_{2}^{\prime}-1 \geqslant$ 1 , and $F_{\max T}\left(S^{\prime}\right) \geqslant \max \left\{10 T_{1}^{\prime}, 9 T_{2}^{\prime}\right\} \geqslant 9=F_{\max T}\left(S^{*}\right)$.) 
However, if we take $k=1$ and $l=1$ we get $\boldsymbol{\beta}^{\prime}=(11$, $10,4,3)$ and $S^{*}$ is no longer optimal since $F_{\max T}^{\prime}\left(S^{*}\right)=$ $4 T_{3}^{*}=12$ and $F_{\max T}^{\prime}(S)=10$ for the LWO schedule $S$ defined by $C(S)=(1,2,3,4)$. Hence the conclusion of optimality is meaningless for $S^{*}$.

Example 15. Suppose that the weights $\beta_{i}, i=1, \ldots, n$, are measured on an interval or ordinal scale. If the objective function is $F_{\max T}$, then in the ncw, cdd, ncpt, ns case, there are instances of the scheduling problem and schedules for which the conclusion of optimality is meaningless.

Proof. Let $n=3, p=(3,2,2), d=2$, and $\boldsymbol{\beta}=(10,2,1)$. Then $C\left(S^{*}\right)=(3,7,5)$ defines an optimal schedule which is not an LWO schedule. (It is straightforward to check that $S^{*}$ is optimal since, for any schedule $S^{\prime}, C_{1}^{\prime} \geqslant 3$, so $F_{\max T}\left(S^{\prime}\right) \geqslant 10 T_{1}^{\prime} \geqslant 10=F_{\max T}\left(S^{*}\right)$.)

However, if we take $k=1$ and $l=5$ we get $\boldsymbol{\beta}^{\prime}=(15,7$, 6) and $S^{*}$ is no longer optimal since $F_{\max T}^{\prime}\left(S^{*}\right)=7 T_{2}^{*}=$ 35 and $F_{\max T}^{\prime}(S)=30$ for the LWO schedule $S$ defined by $C(S)=(3,5,7)$. Hence the conclusion of optimality is meaningless for $S^{*}$.

We conclude the verification of Table I by introducing two principles for generating new examples from old examples.

Principle 1. For $F=F_{\text {sumE } \mid T}, F_{\text {sum } T}, F_{\max E \mid T}$, or $F_{\max T}$, suppose $E$ is an example in a case with cdd for which the conclusion of optimality is meaningless for all optimal schedules $S$ if the weights are measured on an interval or ordinal scale. Furthermore, suppose that there exists an admissible transformation of weights $\Phi, \Phi(x)=k x+l$, $k>0$, such that no optimal schedule $S$ remains optimal after $\Phi$ is applied. Then we can find an example $\bar{E}$ such that ncdd holds and such that the conclusion of optimality is meaningless for all optimal schedules in $\bar{E}$ if the weights are measured on an interval and ordinal scale. Moreover, if $n c p t$ or $s$ or ns holds in $E$, it also holds in $\bar{E}$. However, if $\mathrm{cw}$ holds in $E$, we can define $\vec{E}$ so that cw also holds in $\bar{E}$ or we can define it so that ncw holds in $\bar{E}$. Also, if cpt holds in $E$, we can define $\bar{E}$ so that cpt also holds in $\bar{E}$ or we can define it so that ncpt holds in $\bar{E}$.

Proof. Given example $E$, define $\bar{E}$ by adding a new $(n+$ 1)st job with a very large due date $d_{n+1}$, a processing time $p_{n+1}$ equal to the minimum $p_{i}$ from $E$, and weights $\alpha_{n+1}=$ $\min \alpha_{i}$ and $\beta_{n+1}=\min \beta_{i}$ from $E$. For example, we could take

$d_{n+1}=\max _{i \leqslant n} d_{i}+\sum_{i=1}^{n} p_{i}+\max _{i \leqslant n} p_{i}$.

If $S$ was optimal for $E$, then taking $C_{n+1}=d_{n+1}$ certainly gives an optimal schedule $\bar{S}$ for $\bar{E}$. Also note that the completion times of the first $n$ jobs of any optimal schedule $\bar{U}$ in $\bar{E}$ define an optimal schedule $U$ in $E$, for if $S$ is optimal in $E$ and $\bar{S}$ is defined as above, then if $F$ is any of the penalty functions under consideration,
$F(\bar{U}) \geqslant F(U) \geqslant F(S)=F(\bar{S})=F(\bar{U})$,

so $F(U)=F(S)$. If in $\bar{E}, \bar{U}$ is an optimal schedule, and if $S^{\prime}$ had a lower objective function value than $U$ after a transformation of weights in $E$, then taking $C_{n+1}^{\prime}=d_{n+1}$ gives a schedule $\bar{S}^{\prime}$ for $\bar{E}$ with a lower objective function value than $\bar{U}$ after the same transformation of weights.

Note that $\bar{E}$ has property ncdd. If $E$ had ncpt or ns or s, so does $\bar{E}$. If $E$ had cw, then $\bar{E}$ as constructed has cw. However, we can modify the construction of $\bar{E}$ by changing $\alpha_{n+1}$ and $\beta_{n+1}$ to be some very small numbers, getting an example $\bar{E}$ with ncw. If $E$ had cpt, then $\bar{E}$ as constructed has cpt. However, we can modify $\bar{E}$ by changing $p_{n+1}$ to be some very small amount and get an example $\bar{E}$ with ncpt.

Principle 2. For $F=F_{\max \mid T}$, suppose E. is an example in the case $c w, c d d, c p t, n s$ for which the conclusion of optimality is meaningless for all optimal schedules $S$ if the weights are measured on an interval or ordinal scale. Furthermore, suppose that there exists an admissible transformation of weights $\Phi, \Phi(x)=k x+l, k, l>0$, such that no optimal schedule $S$ remains optimal after $\Phi$ is applied. Then we can find an example $\bar{E}$ such that $n c w, c d d$, and ns hold and such that there is no optimal schedule in $\bar{E}$ for which the conclusion of optimality is meaningful if the weights are measured on an interval or ordinal scale. More over, we can define the example so that cpt also holds in $\bar{E}$ or we can define it so that ncpt holds in $\bar{E}$.

Proof. Given $E$, define $\vec{E}$ by adding an $(n+1)$ st job with $d_{n+1}=d$, and $p_{n+1}=q$, where we use $q=p$ if we want cpt and arbitrary $q \neq p$ otherwise. Choose $\gamma>0$ so that

$\gamma\left|\left(\max _{i \leqslant n} C_{i}\right)+q-d\right|<F_{\max E \mid T}(S)$

for every schedule $S$ that is optimal in $E$ and

$(k \gamma+l)\left|\left(\max _{i \leqslant n} C_{i}^{\prime}\right)+q-d\right|<F_{\max E \mid T}^{\prime}\left(S^{\prime}\right)$

for every schedule $S^{\prime}$ that is optimal in $E$ after the weights are transformed by admissible transformation $\Phi$. Let $\alpha_{n+1}=\beta_{n+1}=\gamma$. Define $\bar{S}$ from $S$ by letting $C_{n+1}=$ $\left(\max _{i \leqslant n} C_{i}\right)+q$ and $\overline{S^{\prime}}$ from $S^{\prime}$ by letting $C_{n+1}^{\prime}=$ $\left(\max _{i \leqslant n} C_{i}^{\prime}\right)+q$. Then by Equation (2),

$F_{\max E \mid T}(\bar{S})=F_{\max E \mid T}(S)$,

and by Equation (3),

$F_{\max E \mid T}^{\prime}\left(\overline{S^{\prime}}\right)=F_{\max E \mid T}^{\prime}\left(S^{\prime}\right)$.

Hence, every optimal schedule $S$ in $E$ defines an optimal schedule $\bar{S}$ in $\bar{E}$, and every schedule $S^{\prime}$ in $E$ that is optimal after applying admissible transformation $\Phi$ leads to a schedule $\bar{S}^{\prime}$ in $\bar{E}$ that is optimal after applying $\Phi$. (Note that $\Phi$ is an admissible transformation of weights in $\vec{E}$ since $\Phi(\gamma)>0$ because $k, l>0$.) 


\section{S130 / Mahadev, Peke $\check{C}$ and Roberts}

For every schedule $\bar{U}$ for the $(n+1)$ jobs problem, let $U$ be $\bar{U}$ restricted to the first $n$ jobs. Note that for any optimal $\bar{U}$ in $\bar{E}, U$ must be optimal in $E$, for if $S$ is optimal for the $n$ jobs problem and $\bar{S}$ is defined as above, we have

$$
\begin{gathered}
F_{\max E \mid T}(\bar{U}) \geqslant F_{\max E \mid T}(U) \geqslant F_{\max E \mid T}(S) \\
=F_{\max E \mid T}(\bar{S})=F_{\max E \mid T}(\bar{U}),
\end{gathered}
$$

so $F_{\max E \mid T}(U)=F_{\max E \mid T}(S)$. Hence, no optimal schedule $\bar{U}$ in $\bar{E}$ remains optimal after the weights are transformed by $\Phi$ since there is no optimal schedule $S$ in $E$ which remains optimal after transforming all the weights by $\Phi$.

\section{DISCUSSION}

We have shown that there are situations in scheduling in which an admissible change of scale can change an optimal solution to one which is not optimal. The idea that one needs to consider such a possibility is the most important message in this paper, more important than the specific examples we give.

If weights are measured on a ratio scale, then conclusions about optimality of a schedule are meaningful under the four objective functions we have considered. However, this is not always true for interval scales or ordinal scales. The results of Table I can be summarized as follows: in the case of common weights, if weights are symmetric or if we only apply penalties for tardiness, then conclusions about optimality are meaningful for all of the objective functions we have considered. In other cases, these conclusions can be meaningless (in the sense that there exist weights such that there is no optimal schedule for which the conclusion of optimality is meaningful), except in the cases ncw, cdd, $\mathrm{cpt}, \mathrm{s}$ for objective function $F_{\mathrm{sum} E \mid T}$ and ncw, cdd, cpt, ns for objective function $F_{\text {sum } T}$. As demonstrated by Theorem 2.7 and Examples 14 and 15, the case of common due dates and non-common weights for objective function $F_{\max }$ is different than all other cases that we have considered. In this case, there always exists an optimal schedule (given by the largest weight order) for which the conclusion of optimality is meaningful. However, there can be other optimal schedules for which the conclusion of optimality is meaningless.

One surprising result is that our conclusions about the meaningfulness of the conclusion of optimality are the same for interval and ordinal scales in all the cases that we have considered. It is not hard to give examples of problems in combinatorial optimization where the conclusion of optimality is meaningful for interval scales but not for ordinal scales. (For instance, this is true for the traveling salesman problem where edge weights represent data measured on an interval scale.) It would be interesting to characterize scheduling problems for which the fact that the conclusion of optimality is meaningful for any choice of weights measured on an interval scale implies that the conclusion of optimality is meaningful for any choice of weights measured on an ordinal scale. This is a topic that will require further research.
This paper leaves room for considerably more analysis. For one thing, we have considered only single machines, rather than multiple machines. Second, we have limited discussion to four specific objective functions. A variety of other objective functions are studied in the literature (many are surveyed by Baker and Scudder 1990). It would be interesting to extend the analysis in this paper to some of these other objective functions as well.

We have not discussed measurement of parameters in our problem other than weights. Specifically, we have not discussed the measurement of desired and scheduled completion times and of processing times. Time is measured on a ratio scale when we talk about durations (we can change from minutes to hours or days or years). A change of time scale would, therefore, not affect the truth or falsity of the conclusion of optimality under the four objective functions discussed here. Even if time were measured on an interval scale, as it would be if we talked about "the year 2000" as opposed to "four years from now (1996)," the conclusion of optimality would still be meaningful under these four objective functions. However, this might no longer be true if we were to use "nonlinear" objective functions. Hence for such objective functions, one should analyze meaningfulness not just under admissible transformations of weights, but also under admissible transformations of times, even if both define ratio scales.

The results of this paper suggest certain specific and other more general policy recommendations. When it comes to single machine scheduling problems in which there is any doubt about the appropriateness of the ratio scale as a model for measuring the penalties for tardy or early arrival, the analyst must be careful to consider the meaningfulness or invariance of the conclusion of optimality. In particular, in certain cases (as summarized in Table I), the analyst should be dubious about any claim of optimality. More generally, the main policy recommendation we make is that operations researchers should be aware of the possible impact of the way that we measure things on the class of optimal solutions to a problem. Before developing or applying methods to compute an optimal solution for any operations research problem, the analyst should think about the types of scales used in measuring parameters involved in the objective function, and should consider whether or not it is meaningful to claim that a given solution is optimal. This warning applies even if the problem and methods are of a standard nature. If the conclusion of optimality might not be meaningful, the analyst should try to reformulate the problem in such a way that he/she can be comfortable that the conclusion of optimality will be meaningful.

\section{APPENDIX}

In this appendix we provide the proofs that $S$ is (uniquely) optimal in the examples where these proofs were omitted.

Example 2. Here $n=3, d=3.1, p=(1,1,1.1), \alpha=1, \beta$ $=10$ and $C(S)=(2.1,3.1,1.1)$. Then 


$$
\begin{aligned}
F_{\text {sumE|T}}(S)= & 1 \cdot(3.1-2.1) \\
& +10(3.1-3.1)+1 \cdot(3.1-1.1)=3 .
\end{aligned}
$$

We show that $S$ is the unique optimal schedule up to symmetry. (Note that jobs 1 and 2 are equivalent, i.e., $d_{1}=$ $d_{2}, p_{1}=p_{2}, \alpha_{1}=\alpha_{2}$, and $\beta_{1}=\beta_{2}$.) Suppose $C\left(S^{\prime}\right)=\left(C_{1}^{\prime}\right.$, $\left.C_{2}^{\prime}, C_{3}^{\prime}\right)$. Furthermore, by symmetry, we assume $C_{1}^{\prime}<C_{2}^{\prime}$. If any $C_{i}^{\prime}>3.4$, then

$F_{\text {sumE|T}}\left(S^{\prime}\right)>10(3.4-3.1)=3=F_{\text {sumE } \mid T}(S)$.

So, we assume that $C_{i}^{\prime} \leqslant 3.4$ for all $i$. We now consider three cases.

Case 1. $C_{1}^{\prime}<C_{2}^{\prime}<C_{3}^{\prime}$. Then since $C_{1}^{\prime}+C_{2}^{\prime}+C_{3}^{\prime} \geqslant p_{1}$ $+p_{2}+p_{3}$, we have $C_{3}^{\prime} \geqslant 3.1$. Suppose it is equal to $3.1+$ $\delta$, for $\delta \geqslant 0$. Since $C_{3}^{\prime} \leqslant 3.4$, we have $\delta \leqslant 3$. Now $C_{2}^{\prime} \leqslant$ $2+\delta$ and $C_{1}^{\prime} \leqslant 1+\delta$. Hence,

$$
\begin{aligned}
F_{\mathrm{sum} E \mid T}\left(S^{\prime}\right) \geqslant & 1 \cdot(3.1-(1+\delta)) \\
& +1 \cdot(3.1-(2+\delta))+10((3.1+\delta)-3.1) \\
= & 3.2+8 \delta \geqslant 3.2>F_{\mathrm{sum} E \mid T}(S) .
\end{aligned}
$$

Case 2. $C_{1}^{\prime}<C_{3}^{\prime}<C_{2}^{\prime}$. Then $C_{2}^{\prime} \geqslant 3.1$; say it is $3.1+\delta$. As in Case 1 , we have $0 \leqslant \delta \leqslant 0.3$. Now $C_{3}^{\prime} \leqslant 2.1+\delta$ and $C_{1}^{\prime} \leqslant 1+\delta$. Hence,

$$
\begin{aligned}
F_{\text {sumE|T}}\left(S^{\prime}\right) \geqslant & 1 \cdot(3.1-(1+\delta)) \\
& +10((3.1+\delta)-3.1)+1 \cdot(3.1-(2.1+\delta)) \\
= & 3.1+8 \delta \geqslant 3.1>F_{\text {sumE } \mid T}(S) .
\end{aligned}
$$

Case 3. $C_{3}^{\prime}<C_{1}^{\prime}<C_{2}^{\prime}$. In this case, as in the other cases, we have $C_{2}^{\prime}=3.1+\delta, 0 \leqslant \delta \leqslant 0.3$, and we have $C_{1}^{\prime}$ $\leqslant 2.1+\delta, C_{3}^{\prime} \leqslant 1.1+\delta$. Then

$$
\begin{aligned}
F_{\text {sumE } \mid T}\left(S^{\prime}\right) \geqslant & 1 \cdot(3.1-(2.1+\delta)) \\
& +10((3.1+\delta)-3.1)+1 \cdot(3.1-(1.1+\delta)) \\
= & 3+8 \delta \geqslant 3 \geqslant F_{\text {sum } E \mid T}(S),
\end{aligned}
$$

and equality holds if and only if $S^{\prime}=S$. We conclude that $S$ is the optimal schedule up to symmetry among the first two jobs. (The other optimal schedule is (3.1, 2.1, 1.1).)

Example 3. Here $n=3, d=2, p=(1,1,1+q)$ with $q=$ 0 or $.01, \boldsymbol{\alpha}=\left(\frac{1}{2}, \frac{1}{3}, 1\right), \boldsymbol{\beta}=\left(\frac{1}{16}, \frac{1}{81}, 1\right)$, and $C(S)=(3,4,2)$. The case $q=0$ of course will give us cpt, while the case $q=0.01$ will give us ncpt. Then if $\lambda=\frac{1}{16}+\frac{2}{81}$,

$F_{\text {sumE|T}}(S)=\frac{1}{16}(3-2)+\frac{1}{81}(4-2)+1 \cdot(2-2)=\lambda$.

Let $C\left(S^{\prime}\right)=\left(C_{1}^{\prime}, C_{2}^{\prime}, C_{3}^{\prime}\right)$. If $C_{3}^{\prime}>2+\lambda$ or $C_{3}^{\prime}<2-\lambda$, then

$F_{\text {sumE } \mid T}\left(S^{\prime}\right)>1 \cdot((2+\lambda)-2)=\lambda=F_{\text {sum } E \mid T}(S)$,

or

$F_{\text {sum } E \mid T}\left(S^{\prime}\right)>1 \cdot(2-(2-\lambda))=\lambda=F_{\text {sumE|T}}(S)$.

Suppose $2-\lambda \leqslant C_{3}^{\prime} \leqslant 2+\lambda$. Observe that $\lambda=\frac{1}{16}+\frac{2}{81}<$ $\frac{1}{4}$. If $C_{1}^{\prime}$ (respectively, $C_{2}^{\prime}$ ) is less than $C_{3}^{\prime}$, then $C_{1}^{\prime}$ (respectively, $C_{2}^{\prime}$ ) is at most $1+\lambda-q$. Hence we have

$$
\begin{aligned}
F_{\text {sum } E \mid T}\left(S^{\prime}\right) & \geqslant \frac{1}{2}(2-(1+\lambda-q)) \\
& \geqslant \frac{1}{2}(1-\lambda)>\lambda=F_{\operatorname{sum} E \mid T}(S),
\end{aligned}
$$

or

$$
\begin{aligned}
F_{\text {sumE|T}}\left(S^{\prime}\right) & \geqslant \frac{1}{3}(2-(1+\lambda-q)) \\
& \geqslant \frac{1}{3}(1-\lambda)>\lambda=F_{\text {sumE } \mid T}(S) .
\end{aligned}
$$

Thus, we may assume that $C_{1}^{\prime}, C_{2}^{\prime}>C_{3}^{\prime}$. If $C_{3}^{\prime}>2$, then $C_{1}^{\prime}$ $>3$ and $C_{2}^{\prime}>4$, or $C_{1}^{\prime}>4$ and $C_{2}^{\prime}>3$. It follows that

$$
\begin{aligned}
F_{\mathrm{sum} E \mid T}\left(S^{\prime}\right) & >\frac{1}{16}(3-2)+\frac{1}{81}(4-2) \\
& =\lambda=F_{\text {sumE|T}}(S)
\end{aligned}
$$

Thus, we may suppose that $C_{3}^{\prime} \leqslant 2$. Then $C_{3}^{\prime}=2-\delta, \delta \geqslant$ 0 . We know $\delta \leqslant \lambda$ since $C_{3}^{\prime} \geqslant 2-\lambda$. It follows that $C_{1}^{\prime} \geqslant$ $3-\delta$ and $C_{2}^{\prime} \geqslant 4-\delta$ or $C_{1}^{\prime} \geqslant 4-\delta$ and $C_{2}^{\prime} \geqslant 3-\delta$. Since $\delta \leqslant \lambda<1 / 4$, both jobs 1 and 2 are tardy. Hence,

$$
\begin{aligned}
F_{\text {sumE } \mid T}\left(S^{\prime}\right) \geqslant & \frac{1}{16}(3-\delta-2)+\frac{1}{81}(4-\delta-2) \\
& +1 \cdot(2-(2-\delta)) \\
= & \frac{1}{16}+\frac{2}{81}+\delta\left(1-\frac{1}{16}-\frac{1}{81}\right) \\
= & \lambda+\delta\left(1-\frac{1}{16}-\frac{1}{81}\right) \\
\geqslant & \lambda=F_{\text {sumE } \mid T}(S)
\end{aligned}
$$

and equality holds if and only if $C\left(S^{\prime}\right)=C(S)=(3,4,2)$. It follows that $S$ is the unique optimal schedule.

Example 5. Here $n=4, \boldsymbol{d}=(2,2,2,1), p=1, \boldsymbol{\alpha}=\boldsymbol{\beta}=$ $(9,9,9,1)$ and $C(S)=(2,1,3,4)$. Then

$$
\begin{aligned}
F_{\text {sum } E \mid T}(S)= & 9(2-2)+9(2-1) \\
& +9(3-2)+1 \cdot(4-1)=21 .
\end{aligned}
$$

Consider $S^{\prime}$ with $C\left(S^{\prime}\right)=\left(C_{1}^{\prime}, C_{2}^{\prime}, C_{3}^{\prime}, C_{4}^{\prime}\right)$. By symmetry, we may assume that $C_{2}^{\prime}<C_{1}^{\prime}<C_{3}^{\prime}$. If $C_{4}^{\prime}<C_{1}^{\prime}$, then $C_{1}^{\prime} \geqslant$ 3 and $C_{3}^{\prime} \geqslant 4$, so

$F_{\text {sumE } \mid T}\left(S^{\prime}\right) \geqslant 9(3-2)+9(4-2)=27>F_{\text {sumE } \mid T}(S)$.

Thus, suppose that $C_{1}^{\prime}<C_{4}^{\prime}$. If $C_{2}^{\prime} \geqslant 2$, then $C_{1}^{\prime} \geqslant 3, C_{3}^{\prime} \geqslant$ 4 and again we obtain

$F_{\text {sum } E \mid T}\left(S^{\prime}\right) \geqslant 9(3-2)+9(4-2)=27>F_{\text {sum } E \mid T}(S)$.

Thus, we may assume that $C_{2}^{\prime}=2-\lambda$, with $\lambda>0$. Note that since $p_{2}=1, \lambda \leqslant 1$. Also, we have $C_{1}^{\prime} \geqslant 3-\lambda$. Moreover, either

Case 1. $C_{3}^{\prime} \geqslant 4-\lambda$ and $C_{4}^{\prime} \geqslant 5-\lambda$ or

Case 2. $C_{3}^{\prime} \geqslant 5-\lambda$ and $C_{4}^{\prime} \geqslant 4-\lambda$. In Case 1,

$$
\begin{aligned}
F_{\text {sum } E \mid T}\left(S^{\prime}\right) \geqslant & 9(2-(2-\lambda))+9((3-\lambda)-2) \\
& +9((4-\lambda)-2)+1 \cdot((5-\lambda)-1) \\
= & 9(3-\lambda)+4-\lambda \\
\geqslant & 9 \cdot 2+3=21=F_{\text {sum } E \mid T}(S),
\end{aligned}
$$


and the equality holds if and only if $\lambda=1$ and $C\left(S^{\prime}\right)=$ $C(S)=(2,1,3,4)$

In Case 2,

$$
\begin{aligned}
F_{\text {sum } E \mid T}\left(S^{\prime}\right) \geqslant & 9(2-(2-\lambda))+9((3-\lambda)-2) \\
& +9((5-\lambda)-2)+1 \cdot((4-\lambda)-1) \\
= & 9(4-\lambda)+3-\lambda \\
\geqslant & 9 \cdot 3+2=29>F_{\text {sumE|T}}(S) .
\end{aligned}
$$

Hence, $S$ is the unique optimal schedule up to symmetry among the first three jobs.

Example 7. Here $n=4, d=(2,2,2,1), p=1, \boldsymbol{\beta}=(9,9$, $9,1)$ and $C(S)=(1,2,3,4)$. Then

$F_{\text {sumT }}(S)=9 \cdot 0+9 \cdot 1+1 \cdot 3=12$.

Consider $C\left(S^{\prime}\right)=\left(C_{1}^{\prime}, C_{2}^{\prime}, C_{3}^{\prime}, C_{4}^{\prime}\right)$. By symmetry, we may assume that $C_{1}^{\prime}<C_{2}^{\prime}<C_{3}^{\prime}$. If $C_{3}^{\prime} \geqslant 4$, then

$$
\begin{aligned}
F_{\text {sum } T}\left(S^{\prime}\right) & \geqslant \beta_{3}\left(C_{3}^{\prime}-2\right) \geqslant \beta_{3}(4-2) \\
& =9 \cdot 2=18>F_{\operatorname{sum} T}(S) .
\end{aligned}
$$

Thus, we may assume that $C_{3}^{\prime}<4$ and hence, since all $p_{i}=$ 1 , we have $C_{4}^{\prime} \geqslant 4$. Also, again since all $p_{i}=1$, we have $C_{1}^{\prime}$ $\geqslant 1, C_{2}^{\prime} \geqslant 2, C_{3}^{\prime} \geqslant 3$. Hence, if $C_{4}^{\prime}=4$, then $S^{\prime}=S$. If $C_{4}^{\prime}$ $>4$, then

$$
\begin{aligned}
F_{\text {sum } T}\left(S^{\prime}\right) & >\beta_{3}(3-2)+\beta_{4}(4-1) \\
& =9(3-2)+1 \cdot(4-1)=12=F_{\text {sum } T}(S) .
\end{aligned}
$$

Thus, $S$ is the unique optimal schedule up to symmetry among the first three jobs.

Example 8. Here $n=3, d=3, p=1, \alpha=1, \beta=10$ and $C(S)=\left(\frac{13}{11}, \frac{24}{11}, \frac{35}{11}\right)$. We have

$$
\begin{aligned}
F_{\max \mid T}(S) & =\max \left\{1 \cdot\left(3-\frac{13}{11}\right), 1 \cdot\left(3-\frac{24}{11}\right), 10\left(\frac{35}{11}-3\right)\right\} \\
& =\frac{20}{11} .
\end{aligned}
$$

To see that $S$ is optimal, consider $C\left(S^{\prime}\right)=\left(C_{1}^{\prime}, C_{2}^{\prime}, C_{3}^{\prime}\right)$. By symmetry, we may assume that $C_{1}^{\prime}<C_{2}^{\prime}<C_{3}^{\prime}$. If $C_{1}^{\prime}<\frac{13}{11}$, then

$F_{\max \mid T}\left(S^{\prime}\right)>1 \cdot\left(3-\frac{13}{11}\right)=\frac{20}{11}=F_{\max E \mid T}(S)$.

Hence, $C_{1}^{\prime} \geqslant \frac{13}{11}$, and thus $C_{3}^{\prime} \geqslant \frac{35}{11}$. If $C_{3}^{\prime}>\frac{35}{11}$, then

$F_{\max E \mid T}\left(S^{\prime}\right)>10 \cdot\left(\frac{35}{11}-3\right)=\frac{20}{11}=F_{\max \mid T}(S)$.

Hence, $C_{3}^{\prime}=\frac{35}{11}, C_{1}^{\prime}=\frac{13}{11}$. It follows that $C_{2}^{\prime}=\frac{24}{11}$, and so $S$ is the unique optimal schedule up to symmetry among all three jobs.

Example 9. Here $n=3, d=3, p=(1,1,1.1), \alpha=1, \beta=$ 10 and $C(S)=\left(\frac{24}{11}, \frac{35}{11}, \frac{13}{11}\right)$. Then

$$
\begin{aligned}
F_{\max E \mid T}(S) & =\max \left\{1 \cdot\left(3-\frac{24}{11}\right), 10\left(\frac{35}{11}-3\right), 1 \cdot\left(3-\frac{13}{11}\right)\right\} \\
& =\frac{20}{11} .
\end{aligned}
$$

To see that $S$ is optimal, consider $S^{\prime}$ with $C\left(S^{\prime}\right)=\left(C_{1}^{\prime}, C_{2}^{\prime}\right.$, $\left.C_{3}^{\prime}\right)$. By symmetry, we may assume that $C_{1}^{\prime}<C_{2}^{\prime}$. If for $i=$ 1,2 , or $3, C_{i}^{\prime}<\frac{13}{11}$, then

$F_{\max E \mid T}\left(S^{\prime}\right)>1 \cdot\left(3-\frac{13}{11}\right)=\frac{20}{11}=F_{\max \mid T}(S)$.

Similarly, if $C_{i}^{\prime}>\frac{35}{11}$, then

$F_{\max \mid T}\left(S^{\prime}\right)>10\left(\frac{35}{11}-3\right)=\frac{20}{11}=F_{\max E \mid T}(S)$.

Hence, we may assume that

$\frac{13}{11} \leqslant C_{i}^{\prime} \leqslant \frac{35}{11}, \quad i=1,2,3$.

If $C_{1}^{\prime}<C_{2}^{\prime}<C_{3}^{\prime}$, then

$C_{3}^{\prime} \geqslant C_{1}^{\prime}+p_{2}+p_{3} \geqslant \frac{13}{11}+2.1=3+\frac{31}{110}$,

so

$F_{\max E \mid T}\left(S^{\prime}\right) \geqslant 10\left(\left(3+\frac{31}{110}\right)-3\right)=\frac{31}{11}>F_{\max E \mid T}(S)$.

If $C_{1}^{\prime}<C_{3}^{\prime}<C_{2}^{\prime}$, then as above,

$C_{2}^{\prime} \geqslant C_{1}^{\prime}+p_{2}+p_{3} \geqslant 3+\frac{31}{110}$,

and again

$F_{\max E \mid T}\left(S^{\prime}\right) \geqslant \frac{31}{11}>F_{\max E \mid T}(S)$.

If $C_{3}^{\prime}<C_{1}^{\prime}<C_{2}^{\prime}$, then

$C_{2}^{\prime} \geqslant C_{3}^{\prime}+p_{1}+p_{2} \geqslant \frac{13}{11}+2=\frac{35}{11}$.

Since $C_{2}^{\prime} \leqslant \frac{35}{11}$ by Equation (4), equality holds in (5), and because $C_{3}^{\prime} \geqslant \frac{13}{11}$ by $(4), C\left(S^{\prime}\right)=C(S)=\left(\frac{24}{11}, \frac{35}{11}, \frac{13}{11}\right)$. We conclude that $S$ is the unique optimal schedule up to symmetry among the first two jobs.

Example 10. Here $n=2, d=2, p=1, \boldsymbol{\alpha}=\boldsymbol{\beta}=(1,2)$, $C(S)=\left(\frac{8}{3}, \frac{5}{3}\right)$ and $C\left(S^{\prime}\right)=\left(\frac{4}{3}, \frac{7}{3}\right)$. Note that

$F_{\max \mid T}(S)=\max \left\{1\left(\frac{8}{3}-2\right), 2\left(2-\frac{5}{3}\right)\right\}=\frac{2}{3}$,

$F_{\max E \mid T}\left(S^{\prime}\right)=\max \left\{1\left(2-\frac{4}{3}\right), 2\left(\frac{7}{3}-2\right)\right\}=\frac{2}{3}$.

Consider $S^{\prime \prime}$ with $C\left(S^{\prime \prime}\right)=\left(C_{1}^{\prime \prime}, C_{2}^{\prime \prime}\right)$.

Case 1. $C_{1}^{\prime \prime}<C_{2}^{\prime \prime}$.

If $C_{1}^{\prime \prime}<\frac{4}{3}$ then

$F_{\max E \mid T}\left(S^{\prime \prime}\right)>\left(2-\frac{4}{3}\right)=\frac{2}{3}=F_{\max E \mid T}\left(S^{\prime}\right)$.

Similarly, if $C_{2}^{\prime \prime}>\frac{7}{3}$, then

$F_{\max E \mid T}\left(S^{\prime \prime}\right)>2\left(\frac{7}{3}-2\right)=\frac{2}{3}=F_{\max E \mid T}\left(S^{\prime}\right)$.

Hence, $S^{\prime \prime}$ is optimal if and only if $S^{\prime \prime}=S^{\prime}$ (because in any optimal schedule, $\frac{4}{3} \leqslant C_{1}^{\prime \prime}<C_{1}^{\prime \prime}+p_{1} \leqslant C_{2}^{\prime \prime} \leqslant \frac{7}{3}$ ). 
Case 2. $C_{1}^{\prime \prime}>C_{2}^{\prime \prime}$.

If $C_{1}^{\prime \prime}>\frac{8}{3}$ then

$F_{\max E \mid T}\left(S^{\prime \prime}\right)>\left(\frac{8}{3}-2\right)=\frac{2}{3}=F_{\max E \mid T}(S)$.

Similarly, if $C_{2}^{\prime \prime}<\frac{5}{3}$, then

$F_{\max \mid T}\left(S^{\prime \prime}\right)>2\left(2-\frac{5}{3}\right)=\frac{2}{3}=F_{\max \mid T}(S)$.

Hence, $S^{\prime \prime}$ is optimal if and only if $S^{\prime \prime}=S$ (because in any optimal schedule, $\frac{5}{3} \leqslant C_{2}^{\prime \prime}<C_{2}^{\prime \prime}+p_{2} \leqslant C_{1}^{\prime \prime} \leqslant \frac{8}{3}$ ). Thus, the only optimal schedules are $S$ and $S^{\prime}$.

Example 11. Here $n=2, d=3, p=(1,2), \boldsymbol{\alpha}=\boldsymbol{\beta}=(1$, $2)$, and $C(S)=\left(\frac{11}{3}, \frac{8}{3}\right)$. Then

$F_{\max \mid T}(S)=\max \left\{1\left(\frac{11}{3}-3\right), 2\left(3-\frac{8}{3}\right)\right\}=\frac{2}{3}$.

Consider $S^{\prime}$ with $C\left(S^{\prime}\right)=\left(C_{1}^{\prime}, C_{2}^{\prime}\right)$.

If $C_{1}^{\prime}<\frac{7}{3}$ then

$F_{\max E \mid T}\left(S^{\prime}\right)>\left(3-\frac{7}{3}\right)=\frac{2}{3}=F_{\max E \mid T}(S)$.

Similarly, if $C_{2}^{\prime}>\frac{10}{3}$, then

$F_{\max E \mid T}\left(S^{\prime}\right)>2\left(\frac{10}{3}-3\right)=\frac{2}{3}=F_{\max E \mid T}(S)$.

Therefore, we may assume that $C_{1}^{\prime}>C_{2}^{\prime}$ (otherwise, $C_{2}^{\prime} \geqslant$ $C_{1}^{\prime}+p_{2} \geqslant \frac{7}{3}+2>\frac{10}{3}$ ).

If $C_{1}^{\prime}>\frac{11^{3}}{3}$ then

$F_{\max \mid T}\left(S^{\prime}\right)>\left(\frac{11}{3}-3\right)=\frac{2}{3}=F_{\max \mid T}(S)$.

Similarly, if $C_{2}^{\prime}<\frac{8}{3}$, then

$F_{\max E \mid T}\left(S^{\prime}\right)>2\left(3-\frac{8}{3}\right)=\frac{2}{3}=F_{\max E \mid T}(S)$.

Hence, $S^{\prime}$ is optimal if and only if $S^{\prime}=S$ (because in any optimal schedule, $\frac{8}{3} \leqslant C_{2}^{\prime}<C_{2}^{\prime}+p_{1} \leqslant C_{1}^{\prime} \leqslant \frac{11}{3}$ ).

\section{ACKNOWLEDGMENTS}

The authors thank the associate editor and the referees for their extremely helpful comments. Aleksandar Pekeč and Fred Roberts thank the National Science Foundation for its support under grant SES-9211492 to Rutgers University. Fred Roberts also thanks the Air Force Office of Scientific Research for its support under grants F49620-931-0041 and F49620-95-1-0233 to Rutgers University.

\section{REFERENCES}

Abdul-RazaQ, T. S. and C. N. Potts. 1988. Dynamic Programming State-Space Relaxation for Single-Machine Scheduling. J. Opnl. Res. Soc. 39, 141-152.

Abdul-RazaQ, T. S., C. N. PotTs, and L. N. van WassenHOVE. 1990. A Survey of Algorithms for the Single Machine Total Weighted Tardiness Scheduling Problem. Discrete Appl. Math. 26, 235-253.

Ahmed, M. U. and P. S. Sundararaghavan. 1990. Minimizing the Weighted Sum of Late and Early Completion Penalties in a Single Machine. IIE Trans. 288-290.
Auguston, K. A. 1989. Parts Delivery System Takes off at O'Hare Int'l Airport. Modern Materials Handling. October, 52-54.

Bagchi, U., Y. Chang, and R. Sullivan. 1987. Minimizing Absolute and Squared Deviations of Completion Times with Different Earliness and Tardiness Penalties and a Common Due Date. Naval Res. Logist. 34, 739-751.

Bagchi, U., R. Sullivan, and Y. Chang. 1986. Minimizing Mean Absolute Deviation of Completion Times About a Common Due Date. Naval Res. Logist. 33, 227-240.

BaKer, K. R. AND G. W. Scudder. 1989. On the Assignment of Optimal Due Dates. J. Opnl. Res. Soc. 40, 93-95.

Baker, K. R. ANd G. W. Scudder. 1990. Sequencing with Earliness and Tardiness Penalties: A Review. Opns. Res. 38, 22-36.

Bector, C., Y. Gupta, AND M. GuPTA. 1988. Determination of an Optimal Due Date and Optimal Sequence in a Single Machine Job Shop. Int. J. Prod. Res. 26, 613-628.

Chand, S. and H. Schneeberger. 1988. Single Machine Scheduling to Minimize Weighted Earliness Subject to No Tardy Jobs. European J. Opnl. Res. 34, 221-230.

Cheng, T. 1987. An Algorithm for the CON Due Date Determination and Sequencing Problem. Comp. Opns. Res. 14, 537-542.

Cheng, T. C. E. 1990. Dynamic Programming Approach to the Single-Machine Sequencing Problem with Different Due-Dates. Computers Math. Applic. 19, 1-7.

CoOmBs, C. H., R. M. DawES, AND A. TversKy. 1970. Mathematical Psychology: An Elementary Introduction. PrenticeHall, Englewood Cliffs, NJ.

Cozzens, M. AND F. S. RoBERTs. 1991. Greedy Algorithms for $T$-Colorings of Graphs and the Meaningfulness of Conclusions about Them. J. Comb. Inf. and Syst. Sci. 16, 286-299.

Elsayed, E. A., M.-K. Lee, S. Kim, ANd E. Scherer. 1993. Sequencing and Batching Procedures for Minimizing Earliness and Tardiness Penalty of Order Retrievals. Int. J. Prod. Res. 31, 727-738.

Emmons, H. 1987. Scheduling to a Common Due Date on Parallel Common Processors. Naval Res. Logist. 34, 803-810.

FERris, M. C. AND M. VLACH. 1992. Scheduling with Earliness and Tardiness Penalties. Naval Res. Logist. 39, 229-245.

Fry, T., R. D. Armstrong, and J. H. Blackstone. 1987 a. Minimizing Weighted Absolute Deviation in Single Machine Scheduling. IIE Trans. 19, 445-450.

FRY, T. D. AND G. LEONG. 1987. A Bi-Criterion Approach to Minimizing Inventory Costs on a Single Machine When Early Shipments are Forbidden. Comp. Opns. Res. 14, 363-368.

Fry, T., G. LeONG, and R. RaKes. 1987b. Single Machine Scheduling: A Comparison of Two Solution Procedures. Omega, 15, 277-282.

HaLl, N. 1986. Single and Multi-Processor Models for Minimizing Completion Time Variance. Naval Res. Logist. 33, $49-54$.

Hall, N. G., W. Kubiak, and S. P. Sethi. 1991. EarlinessTardiness Scheduling Problems, II: Deviation of Completion Times About a Restrictive Common Due Date. Opns. Res. 39, 847-856. 
HALl, N. G. AND M. E. Posner. 1991. Earliness-Tardiness Scheduling Problems I: Weighted Deviation of Completion Times About a Common Due Date. Opns. Res. 39, 836-846.

Hoogeveen, J. A. And S. L. van de Velde. 1991. Scheduling Around a Small Common Due Date. European J. Opnl. Res. 55, 237-242.

KANET, J. J. 1981. Minimizing the Average Deviation of Job Completion Times about a Common Due Date. Naval Res. Logist. 28, 643-651.

KeEney, R. L. AND H. RaIfFA. 1976. Decisions with Multiple Objectives: Preferences and Value Tradeoffs. Wiley, New York.

Koulamas, C. 1994. The Total Tardiness Problem: Review and Extensions. Opns. Res. 42, 1025-1041.

Krantz, D. H., R. D. LuCE, P. Suppes, AND A. TVERSKy. 1971. Foundations of Measurement, Volume I. Academic Press, New York.

Liu, W.-P. And J. B. Sidney. 1996a. Bin Packing Using Semiordinal Data. O. R. Lett. 19, 101-104.

LIU, W.-P. AND J. B. SidNeY. 1996b. Ordinal Algorithms for Packing with Target Center of Gravity. Order, 13, 17-31.

LiU, W.-P., J. B. Sidney, AND A. VAN Vliet. 1996. Ordinal Algorithms for Parallel Machine Scheduling. O. R. Lett. 18, 223-232.

Luce, R. D., D. H. Krantz, P. Suppes, AND A. Tversky. 1990. Foundations of Measurement, Volume III. Academic Press, New York.

Ow, P. S. AND T. E. MorTon. 1988. Filtered Beam Search in Scheduling. Int. J. Prod. Res. 26, 35-62.

Ow, P. S. and T. E. Morton. 1989. The Single Machine Early/Tardy Problem. Mgmt. Sci. 35, 177-191.

Panwalkar, S., M. Smith, AND A. Seidmann. 1982. Common Due Date Assignment to Minimize Total Penalty for the One Machine Scheduling Problem. Opns. Res. 30, 391-399.
Quaddus, M. 1987. A Generalized Model of Optimal DueDate Assignment by Linear Programming. J. Opnl. Res. Soc. 38, 353-359.

RoBERTS, F. S. 1979. Measurement Theory with Applications to Decisionmaking, Utility and the Social Sciences. AddisonWesley, Reading, MA

RoBerts, F. S. 1985. Applications of the Theory of Meaningfulness to Psychology. J. Math. Psychol. 29, 311-332.

Roberts, F. S. 1990. Meaningfulness of Conclusions from Combinatorial Optimization. Discr. Appl. Math. 29, 221-241.

ROBERTS, F. S. 1994. Limitations on Conclusions Using Scales of Measurement. In Operations Research and the Public Sector. A. Barnett, S. M. Pollock, and M. H. Rothkopf (eds.), Elsevier, Amsterdam, 621-671.

Sidney, J. 1977. Optimal Single-Machine Scheduling with Earliness and Tardiness Penalties. Opns. Res. 25, 62-69.

SMITH, W. E. 1956. Various Optimizers for Single-stage Production. Naval Res. Logist. 3, 59-66.

SteVENS, S. S. 1946. On the Theory of Scales of Measurement. Science, 103, 677-680.

Stevens, S. S. 1951. Mathematics, Measurement, and Psychophysics. In Handbook of Experimental Psychology. S. S. Stevens (ed.), Wiley, New York, 1-49.

Stevens, S. S. 1959. Measurement, Psychophysics, and Utility. In Measurement: Definitions and Theories. C. W. Churchman and P. Ratoosh (eds.), Wiley, New York, 18-63.

Sundararaghavan, P. S. And M. U. Ahmed. 1984. Minimizing the Sum of Absolute Lateness in Single-Machine and Multimachine Scheduling. Naval Res. Logist. 31, 325-333.

Suppes, P., D. H. KRaNTZ, R. D. LuCE, AND A. TVERSKY. 1989. Foundations of Measurement, Volume II, Academic Press, New York.

SzwarC, W. 1989. Single Machine Scheduling to Minimize Absolute Deviation of Completion Times from a Common Due Date. Naval Res. Logist. 36, 663-673. 
Copyright 1998, by INFORMS, all rights reserved. Copyright of Operations Research is the property of INFORMS: Institute for Operations Research and its content may not be copied or emailed to multiple sites or posted to a listserv without the copyright holder's express written permission. However, users may print, download, or email articles for individual use. 